\title{
A Novel Family of Quadrupole-Orbitrap Mass Spectrometers for a Broad Range of Analytical Applications
}

Jan-Peter Hauschild ${ }^{1}$, Amelia Peterson ${ }^{1}$, Erik Couzijn ${ }^{1}$, Eduard Denisov ${ }^{1}$, Denis Chernyshev ${ }^{1}$, Christian Hock ${ }^{1}$, Hamish Stewart ${ }^{1}$, Ralf Hartmer ${ }^{1}$, Dmitry Grinfeld ${ }^{1}$, Christian Thoeing ${ }^{1}$, Bastian Reitemeier ${ }^{1}$, Arne Kreutzmann', Oliver Lange ${ }^{1}$, Wilko Balschun ${ }^{1}$, Aivaras Venckus ${ }^{1}$, Sebastian Kanngiesser $^{1}$, Alexander Kholomeev ${ }^{1}$, Gregor Quiring ${ }^{1}$, Frank Czemper ${ }^{1}$, Eloy Wouters ${ }^{2}$, Michael Belford $^{2}$, Mathias Mueller ${ }^{1}$, Jens Grote ${ }^{1}$, Tabiwang N Arrey ${ }^{1}$, Julia Kraegenbring ${ }^{1}$, Kerstin Strupat ${ }^{1}$, Markus Kellmann ${ }^{1}$, Siegrun Mohring ${ }^{1}$, Catharina Crone ${ }^{1}$, Alexander Harder ${ }^{1}$, Aaron M. Robitaille ${ }^{2}$, Khatereh Motamedchaboki², Yang Liu², Aaron S. Gajadhar², Daniel Lopez Ferrer², Eugen Damoc', Angela Criscuolo ${ }^{1}$, Kristina Srzentic ${ }^{2}$, Ed George ${ }^{2}$, Charles Yang ${ }^{2}$, Ioanna Ntai², Amanda Souza ${ }^{2}$, Andreas Wieghaus ${ }^{1}$, Alexander Makarov ${ }^{1 *}$

1- Thermo Fisher Scientific, Bremen, Germany

2- Thermo Fisher Scientific, San Jose, CA, USA

\section{${ }^{*}$ CORRESPONDING AUTHOR}

Prof. Alexander A. Makarov

Thermo Fisher Scientific (Bremen) $\mathrm{GmbH}$

Hanna-Kunath-Str. 11

28199 Bremen, Germany

Tel: $\quad+49(0) 421-5493-410$

Fax: $+49(0) 421-5493-426$

E-Mail: alexander.makarov@thermofisher.com 


\section{ABSTRACT}

The rapidly increasing adoption of high-resolution accurate-mass methods in analytical laboratories has fueled demand for instruments that combine high performance and reliability with small size and greater ease-of-use. This paper presents the major design principles that are driving the evolution of the hybrid quadrupole-Orbitrap instrument architecture to enable a greater range of applications and users. These principles may be summarized as follows: better usage of physical space and better access for service by means of size reduction of pumping and ion optics; expanded use of technologies from electronics in ion-optical design; flexibility in performance via modularity of design of the hardware and software components; and, harmonization of interfaces with other instruments to facilitate sharing and transferability of analytical workflows. The design of a novel family of hybrid mass spectrometers is described in detail, and performance evaluation is carried out on a wide variety of samples for its three representatives: the Orbitrap Exploris 120, Orbitrap Exploris 240 and Orbitrap Exploris 480 mass spectrometers.

The new instrument family is shown to offer compelling potential not only for high-end proteomics and biopharmaceutical applications, but also for screening, trace, targeted and clinical analysis by liquid chromatography/mass spectrometry methods. 


\section{INTRODUCTION}

Mass spectrometry has long established itself as a universal analytical technique for a breathtaking range of applications, spanning from trace elemental, environmental, food, anti-doping and forensic analysis, to analysis of proteins, biopharmaceuticals and even intact viruses. While benefiting greatly from innovations in separation, ionization and data processing technologies, mass spectrometry instrumentation has also undergone multiple fundamental changes in mass analyzer technology and instrument architectures that enabled it to repeatedly expand its analytical power and application reach. One of the key trends of the last two decades is the ever-accelerating transition to highresolution accurate-mass (HRAM) analysis.

Orbitrap $^{\mathrm{TM}}$ mass spectrometry [1] became one of the main beneficiaries and drivers of this trend, with the first commercial instrument, the LTQ Orbitrap ${ }^{\mathrm{TM}}$ mass spectrometer, entering the field in 2005; this instrument has the Orbitrap analyzer acting as an accurate mass detector at the end of a fullyfeatured linear trap instrument [2]. As this LC-compatible family of instruments rapidly progressed [35] towards its ultimate representative, the Orbitrap Elite ${ }^{\mathrm{TM}}$ mass spectrometer (MS) in 2011 [6], another family of simpler, benchtop Orbitrap-only instruments was unveiled in 2008 under the name Exactive $^{\mathrm{TM}}$ [7]. Once equipped with a quadrupole mass filter in 2011 [8], this new Q Exactive ${ }^{\mathrm{TM}}$ family experienced explosive growth both in the scale and variety of applications, its top-end models being the Q Exactive HF in 2014 [9] and the Q Exactive HF-X in 2017 [10] for LC/MS and the Q Exactive GC in 2015 for GC/MS analysis.

Meanwhile, the Orbitrap Elite instrument was superseded for high-end proteomics, pharma and biopharma $\mathrm{MS}^{\mathrm{n}}$ applications by a third family of instruments named the Tribrid ${ }^{\mathrm{TM}}$ platform to reflect the synergy of three analyzers: a hyperbolic-rod quadrupole mass filter, an Orbitrap analyzer, and a dual-pressure linear trap analyzer [11]. Starting with the Orbitrap Fusion ${ }^{\mathrm{TM}}$ MS in 2013 and the Orbitrap Fusion Lumos ${ }^{\mathrm{TM}}$ MS in 2015, this family has also included since 2018 the Orbitrap ID- $X^{\mathrm{TM}}$ MS for small-molecule analysis and since 2019 the Orbitrap Eclipse ${ }^{\mathrm{TM}}$ MS for quantitative proteomics, top-down and native analysis.

This rapid succession of instrument models not only brought continuous improvement of analytical performance, but also facilitated a better appreciation of the fast-expanding demands imposed by modern analytical sciences on MS instrumentation. This has become an inspiration for the development of a next-generation quadrupole-Orbitrap instrument family. Starting from first principles, the authors have aspired to a complete re-thinking of this architecture to enable a more compact, robust, easier to use/service, and higher-performing range of instruments. 


\section{EXPERIMENTAL SECTION.}

All data was generated using Thermo Scientific ${ }^{\mathrm{TM}}$ Orbitrap Exploris ${ }^{\mathrm{TM}} 120$, Orbitrap Exploris 240 , or Orbitrap Exploris 480 mass spectrometers (Thermo Fisher Scientific, Bremen, Germany), as indicated below. Unless specified otherwise, performance is similar across all models.

Initial calibration of all instruments was performed using Thermo Scientific ${ }^{\mathrm{TM}}$ Pierce $^{\mathrm{TM}} \mathrm{FlexMix}^{\mathrm{TM}}$ calibration solution, a mixture of 16 highly pure, ionizable components (mass ranges: 40 to $3000 \mathrm{~m} / \mathrm{z}$ ) developed for both positive and negative ionization calibration.

For pesticide samples, Thermo Scientific ${ }^{\mathrm{TM}}$ HyperSep $^{\mathrm{TM}}$ Dispersive Solid Phase Extraction clean-up products were used for a simplified QuEChERS extraction protocol, with $1 \mu \mathrm{L}$ of sample injected at $300 \mu \mathrm{L} /$ min flow rate using a Thermo Scientific ${ }^{\mathrm{TM}}$ Vanquish Flex Binary ultra-high-performance liquid chromatograph (UHPLC) with Accucore ${ }^{\mathrm{TM}} \mathrm{aQ}$ C18 column $(2.1 \mathrm{~mm} \mathrm{ID} \times 100 \mathrm{~mm}, 2.6 \mu \mathrm{m}$ particles) sustained at $30^{\circ} \mathrm{C}$. Thermo Scientific ${ }^{\mathrm{TM}}$ TraceFinder $^{\mathrm{TM}} 5.1$ software and mzCloud Offline Spectral Library for mzVault 2.3 (Pest_Herb_2020A) was used for processing of targeted analysis data and Thermo Scientific ${ }^{\mathrm{TM}}$ Compound Discoverer ${ }^{\mathrm{TM}}$ 3.1- for processing of untargeted data.

Thermo Scientific ${ }^{\text {TM }}$ TFP-200 VetDrug Explorer Standard mix and TFP-500 VetDrugs Explorer QC Mix were analyzed on the same UHPLC using Acclaim ${ }^{\text {TM }}$ Vanquish PA II $(2.1 \mathrm{~mm}$ ID $\times 150 \mathrm{~mm}, 2.2$ $\mu \mathrm{m}$ particles) column at $40^{\circ} \mathrm{C}$.

Metabolomic analysis was carried out for NIST SRM1950 human plasma standard with 15 min gradient elution using a Thermo Scientific ${ }^{\text {TM }}$ Hypersil GOLD ${ }^{\text {TM }}$ column $(2.1 \mathrm{~mm} \mathrm{ID} \times 150 \mathrm{~mm}, 1.9 \mu \mathrm{m}$ particle size) at a flow rate of $300 \mu \mathrm{L} / \mathrm{min}$ in line with a Thermo Scientific ${ }^{\text {TM }}$ Vanquish ${ }^{\text {TM }}$ Horizon UHPLC system. Data were processed using Compound Discoverer software for unbiased peak detection and identification.

Benchmarking experiments for trastuzumab (Herceptin $($ ) monoclonal antibody were carried out using the Vanquish Horizon UHPLC with a MabPac SEC-1 $(4.0 \mathrm{~mm} \mathrm{ID} \times 150 \mathrm{~mm})$ column for native analysis and a MAbPac RP $(2.1 \mathrm{~mm} I \mathrm{ID} \times 100 \mathrm{~mm}, 4 \mu \mathrm{m}$ particles $)$ column for intact and sub-unit analysis. Digestion to subunits was performed using FabRICATOR® (IdeS) enzyme (Genovis AB, Lund, Sweden). Data were processed with Thermo Scientific ${ }^{\text {TM }}$ BioPharma Finder ${ }^{\text {TM }} 4.0$ software using ReSpect and Xtract intact mass deconvolution algorithms (for isotopically unresolved and resolved spectra, respectively).

Proteomic benchmarking experiments were carried out on Thermo Scientific ${ }^{\mathrm{TM}}$ Pierce $^{\mathrm{TM}} \mathrm{HeLa}$ Protein Digest Standard using the Thermo Scientific ${ }^{\mathrm{TM}}$ EASY-nLC ${ }^{\mathrm{TM}} 1200$ UHPLC system with Thermo Scientific ${ }^{\mathrm{TM}}$ EASY-Spray ${ }^{\mathrm{TM}}$ source and ES803A column. Alternatively, the lonopticks Aurora series UHPLC Column $(75 \mu \mathrm{m} \mathrm{ID} \times 250 \mathrm{~mm})$ with the Thermo Scientific ${ }^{\mathrm{TM}}$ Nanospray Flex ${ }^{\mathrm{TM}}$ ion source was employed. Spectral library search was carried out using Thermo Scientific ${ }^{\mathrm{TM}}$ Proteome Discoverer $^{\mathrm{TM}}$ 2.4 software for data-dependent acquisition experiments and Spectronaut 14 software (Biognosys, Zurich, Switzerland) for data-independent acquisition experiments. In all experiments a false discovery rate of $1 \%$ was applied for both peptides and proteins. 


\section{RESULTS AND DISCUSSION}

\section{Main Principles of the Instrument Architecture}

The new family of mass spectrometers was designed following several general principles. The first principle is the modularity of the design, wherein each module underwent - somewhat paradoxically deeper integration into a self-contained unit.

The instrument presents a user with an LC-compatible ion source connected to an atmosphere-tovacuum interface. In this work, two variants of this module are discussed in detail: the first includes a round-bore metal transfer tube followed by an S-lens, while the second includes a high-capacity transfer tube (HCTT) with an electrodynamic ion funnel. Each interface is fully compatible with any of the ion sources from the Tribrid and TSQ triple quadrupole mass spectrometers. This commonality with the latest Tribrid and TSQ instruments was another major design principle of the new family and can be observed in many aspects of its operation. On the source side, this allows interfacing to the same Tribrid front-end options, such as High-Field Asymmetric Waveform lon Mobility Spectrometry (embodied in the FAIMS Pro ${ }^{\text {TM }}$ interface) or the EASY-IC ${ }^{\text {TM }}$ internal lock-mass ion source.

Following the source module, the analyzer super-module forms the heart of the instrument. It contains several modules: an S-shaped bent flatapole, a hyperbolic-rod segmented quadrupole mass filter, an Independent Charge Detector (ICD) module, a C-trap/Orbitrap block with an ultra-high field Orbitrap mass analyzer and finally an lon-Routing Multipole (IRM) module. All modules are aligned along a common axis and are easily accessible from the top of the chamber (Figure 1). One of the striking features of this super-module is the extensive use of printed-circuit boards (PCBs) as vacuum feedthroughs with predominantly spring-loaded connections, and also as elements of ion optics. Usage of PCBs results in the elimination of tens of cables, -which in turn removes one of the major reasons for electrical failures.

The compact design of the analyzer super-module is directly enabled by one of the most important technical breakthroughs realized in this development: the turbomolecular pump (TMP) module. In all previous families of Orbitrap instruments, the built-in requirement of ultra-high vacuum (UHV) in the $10^{-10}$ or even $10^{-11}$ mbar range for Orbitrap analysis has necessitated a bulky pumping system with several pumps, and this imposed the major constraint on overall design and size of the instrument. In the new instrument, a single, purpose-developed, six-stage TMP evacuates the entire analyzer supermodule. This design results in a drastic reduction of instrument footprint and volume, while alignment of all ion-optical components along a common axis allows for easier access from the sides for electrical connections and from above for servicing. Overall, ease of access and service were primary considerations at every step of the design process.

The analyzer resides within a sheet-metal frame and is contacted from all sides by easy-to-remove electronic boards with spring-loaded contacts. These vertical boards are plugged via PCle connectors into a horizontal base-plane board at the bottom of the instrument, which provides the connections to power as well as internal and external communication. This electronic module embodies the next 
design principle: extensive on-board intelligence combined with both remote and internal diagnostic capabilities.

It should be noted that both analyzer and frame allow access from the rear of the mass spectrometer for any potential extensions of the instrument through the IRM in the future.

Detailed design and operation description for this novel family of high-performance, fit-for-purpose quadrupole-Orbitrap instruments is presented in Section 1 of Supplemental Information. This includes the Orbitrap Exploris $120 \mathrm{MS}$, optimized for quantitative environmental, food safety, and toxicology analysis, as well as other small-molecule applications, and the universal Orbitrap Exploris 240 and 480 MS systems, delivering enhanced performance also for-omics, pharmaceutical and biopharmaceutical applications. Performance characterization of all Orbitrap Exploris models was conducted for their most relevant applications, with selected results presented below.

\section{Stability of Mass Accuracy}

One of the most important characteristics of the Orbitrap mass analyzer since its inception is its high mass accuracy and mass stability. This figure-of-merit is confirmed as shown in Figure 2 for continuously repeated analysis of VetDrug samples using the Orbitrap Exploris 120 instrument, with a subset of 16 substances in a mass range $\mathrm{m} / \mathrm{z} 160$ to 900 evaluated for mass accuracy. While mass stability following system calibration is specified for 24 hours, in standard air-conditioned laboratories measured masses usually remain accurate for much longer, e.g., for several days as shown in Figure $2 a$.

Full scan MS analysis with scan-by-scan internal calibration, using the integrated EASY-IC source to produce the lock-mass ion, was performed to assess the achievable mass accuracy and duration of stable mass accuracy. While adding an overhead of only $9 \mathrm{~ms}$ per scan, this measure maintains mass stability for an extended period (five days or more under appropriate conditions, shown for 15 days) with mass deviations within $1 \mathrm{ppm}$ FWHM (Figure 2b). Stability of the ion signal over the duration of the experiment is illustrated by Figure S-6.

\section{Ultra-high Mass Resolution and Fine Isotopic Structure for Elemental Composition Determination}

Another distinctive feature of Orbitrap mass spectrometry is the ease of obtaining high resolution mass spectra. The importance of ultra-high resolution (>100,000 at $200 \mathrm{~m} / \mathrm{z}$ ) for small molecule analysis is illustrated by Figure 3: already the highest resolution of the lowest-tier instrument Orbitrap Exploris 120 MS (corresponding to 120,000 resolution setting in the Figure) is sufficient to separate the fine isotopic structure of biotin at $\mathrm{m} / \mathrm{z} 244$ and 245 . This drastically reduces the number of possible compounds with the same accurate mass of the main isotope $\mathrm{A} 0$ and also enables, for example, direct tracing of deuterium labeled compounds in stable isotope labeling experiments. At the 240,000 maximum resolution supported by the Orbitrap Exploris $240 \mathrm{MS}$, this fine structure is 
baseline-resolved. The even higher resolution of the Orbitrap Exploris 480 MS pushes the limit of such baseline resolution to significantly higher $\mathrm{m} / \mathrm{z}$.

\section{Scan-to-scan Polarity Switching}

The acquisition scan rate with scan-to-scan polarity switching was accelerated, relative to the $Q$ Exactive instruments, by reducing settling times of ion optics. Shown in Figure 4, a full MS $(30,000$ resolution) and targeted MS/MS (15,000 resolution) in each positive and negative mode are acquired. The dual polarity MS and MS/MS cycle took an average of less than 0.6 seconds (Fig. $4 a$ ). The precursor mass error stayed in low ppm for both positive and negative modes even with external mass calibration, demonstrating that high mass accuracy is retained during polarity switching (Fig. 4b and 4c). The fragment ions in the targeted MS/MS also demonstrated low-ppm mass accuracy (data not shown).

\section{Quantitative Performance on the Example of Pesticide Analysis}

Figure 5 illustrates system performance for pesticide analysis in olive oil, with over 500 pesticides of different classes eluting within less than $10 \mathrm{~min}$ and analyzed in both polarities by an Orbitrap Exploris 240 MS. Each full scan at 45,000 resolution was followed by data-independent acquisition (DIA) scans with $\mathrm{m} / \mathrm{z} 200$-wide windows at 15,000 resolution. Analytes of interest were extracted within a mass window of $2 \mathrm{ppm}$. Part-per-billion ( $\mathrm{ppb}$ or $\mu \mathrm{g} / \mathrm{kg}$ ) and even sub-ppb detection limits were achieved for these pesticides, with two characteristic examples presented in Figure S-7. Substantially identical performance can also be obtained also on the Orbitrap Exploris 120 MS.

\section{Deep Analysis for Metabolomics Samples}

For untargeted metabolomics, AcquireX Deep Scan intelligent acquisition is enabled on the Orbitrap Exploris 240 and $480 \mathrm{MS}$ to automatically exclude background ions and focus MS/MS acquisition on true sample components. Iterative fragmentation with automatically updated inclusion and exclusion lists allows for exhaustive fragmentation deeper into the metabolome as illustrated by Figure 6 for LC/MS of NIST SRM1950 human plasma standard. When compared to the traditional data-dependent acquisition (DDA) analysis of Figure 6a, the number of compounds without MS/MS spectra is reduced by a factor of 3 in Figure 6b. AcquireX acquisition can similarly be employed on the Orbitrap Exploris $480 \mathrm{MS}$. The ability to transfer methods between instruments of this family is also made possible by consistent fragmentation spectra even with different front-end configurations as confirmed by Figure S-8.

Concluding this short tour through the small molecule mode, it should be mentioned that the Orbitrap Exploris family also provides an extension of this mode for fragile metabolites. With the help of mild trapping, the ion energy in the C-trap/IRM region is reduced to strongly decrease precursor fragmentation in full scan mode (Figure S-9). 


\section{Multi-level Biopharmaceutical Characterization}

On the opposite end of the mass range, biopharmaceutical analysis on the Orbitrap Exploris 240 and Orbitrap Exploris $480 \mathrm{MS}$ is facilitated by the Biopharma option. The latter comprises the extension of mass range and usage of optimized system parameters, including the modified pressure settings as described in Section 1 of Supplementary Information. The extended mass range allows analysis of intact antibodies and their conjugates both under native (Figure 7a) and denatured (Figure 7b) conditions. As the protein is not unfolded in the native state, unlike under denaturing conditions, the charge states are significantly lower and thus the antibody is detected at a significantly higher $\mathrm{m} / \mathrm{z}$ range. The full charge state envelopes for the native and denatured intact antibodies are visible in the 30,000-resolution spectra and the glycosylated forms are clearly distinguishable (insets, Figures $7 a$ and $7 \mathrm{~b}$, respectively). The deconvoluted spectra of the native and denatured measurements show low ppm mass errors and almost identical glycan profiles (Figure 7c). Although performance is similar, an important distinction between the experiments is the ability to detect non-covalent complexes under native conditions.

Further subunit analysis following IdeS digestion is possible with baseline isotopic resolution for all subunits and low-ppm mass accuracy (Figure 8). Top-down analysis using HCD fragmentation in the IRM at different collision energies results in cleavage of $58 \%$ residues in the light chain, $42 \%$ residues in Fc/2 and 52\%- in the Fd' subunit (Figure S-10). On the Orbitrap Exploris $480 \mathrm{MS}$, isotopically resolved measurements of the entire heavy chain and its top-down analysis are also possible as shown in Figure S-11.

\section{Performance in Bottom-up Proteomics}

With proteomics becoming one of the first success stories of Orbitrap mass spectrometry following its emergence in 2005 , experiments with proteomic samples present a good opportunity to characterize the system over a very high number and dynamic range of analytes. For proteomic analysis on both Orbitrap Exploris 240 and 480 MS, high reproducibility between technical replicates (Figure S-12) and even between different instruments in different locations worldwide (Figure S-13) was demonstrated. Initial results for tandem mass tag quantitation in short gradients were presented in [12].

Figure 9 presents a comparative evaluation of the Orbitrap Exploris 240 and Orbitrap Exploris 480 systems with the FAIMS Pro interface [13] for DDA analysis of different loads of HeLa Protein Digest Standard using a $90 \mathrm{~min}$ gradient on an Aurora series column (104 min total experiment time). Both instruments demonstrate very high performance, but the higher transmission of the high capacity transfer tube gives a clear advantage to the Orbitrap Exploris 480 MS at low sample loads: almost a tripling of peptide identifications and a doubling of protein identifications at $10 \mathrm{ng}$ are observed. In addition, these data demonstrate a high linear dynamic range of label-free quantitation using the Orbitrap Exploris mass spectrometers (Figure S-14). 
As the DIA paradigm evolved over the last years to deliver increased accuracy, quantitative precision as well as proteome coverage with improved throughput, its requirements for higher ion loads in broader isolation windows appeared to be really well suited to the high space charge capacity and increased speed of the C-trap/Orbitrap combination, as illustrated in [12] for very short ( $<30 \mathrm{~min})$ gradients with the Orbitrap Exploris $480 \mathrm{MS}$.

One of the general hurdles to wider adoption of DIA is the need for cumbersome project-specific library creation. Recently, promising new approaches have been proposed and tested to overcome this limitation utilizing deep neural networks in DIA library creation. The library created in-silico by, e.g., the PROSIT engine is adjusted with the help of a limited number of gas-phase fractionated DIA runs as described in [14-15]. As the result, the Orbitrap Exploris 240 instrument, even without the FAIMS Pro interface, demonstrates very good DIA performance with more than 8,000 proteins identified for $1 \mu \mathrm{g} \mathrm{HeLa} \mathrm{digest} \mathrm{sample} \mathrm{using} 90$ min gradient (Figure 10). Over 9,200 proteins were identified for a mixed sample containing $10 \%$ of E.coli digest.

\section{CONCLUSION}

This work has demonstrated that the new Orbitrap Exploris family of mass spectrometers allows to improve performance and usability of high-resolution accurate mass analysis not only for high-end proteomic applications but also for high-throughput screening, trace and targeted quantitative analysis by LC/MS and LC/MS/MS. Moreover, its performance and modularity offer high potential for expanding this family to new ion sources and applications, such as a mass detector for gas chromatography, a mobile unit for in-situ process analysis and others. 


\section{Acknowledgements}

Part of this work has been funded as part of the MSmed project that has received funding from the European Union's Horizon 2020 research program under grant agreement no. 686547. Part of the work on the Biopharma option has received funding from the European Union's Horizon 2020 research program under grant agreement No. 829157 (TopSpec).

We thank our colleagues at Thermo Fisher Scientific in Bremen and San Jose sites: Stevan Horning, August Specht, Jean-Jacques Dunyach, lain Mylchreest, Thomas Moehring, Ken Miller, Andreas Huhmer, Rajesh Raina, Lisa Thomas, Maciej Bromirski, Shannon Eliuk, Evett Kruka, Mitch Kennedy, Jacob Gudbrand, Prem Singh, Joerg Behrens, Stefan Bruns, Dennie Kemker, David Harmon, Emile de Leeuw, Bjoern Rose, Kyle Fort, Eric Wapelhorst, Michael Antonczak, Sascha Moehring, Jochen Schmidt, Frederik Busse, Hoa Pham.

\section{References}

1. Makarov, A. A. Anal. Chem. 2000, 72, 1156.

2. Makarov, A.; Denisov, E.; Kholomeev, A.; Balschun, W.; Lange, O.; Strupat, K.; Horning, S. Anal. Chem. 2006, 78, 2113.

3. Olsen, J. V.; Macek, B.; Lange, O.; Makarov, A.; Horning, S.; Mann, M. Nat. Methods, 2007, 4, 709.

4. McAlister, G.; Berggren, W.; Griep-Raming, J.; Horning, S.; Makarov, A.; Phanstiel, D.; Stafford, G.; Swaney, D.; Syka, J.; Zabrouskov, V.; Coon, J. J. Proteome Res., 2008, 7, 3127.

5. Olsen, J. V.; Schwartz, J. C.; Griep-Raming, J.; Nielsen, M. L.; Damoc, E.; Denisov, E.; Lange, O.; Remes, P.; Taylor, D.; Splendore, M.; Wouters, E. R.; Senko, M.; Makarov, A.; Mann, M.; Horning, S. Mol. Cell. Proteomics, 2009, 8, 2759.

6. Michalski, A.; Damoc, E.; Lange, O.; Denisov, E.; Nolting, D.; Mueller, M.; Viner, R.; Schwartz, J-; Remes, P.; Belford, M.; Dunyach, J.J.; Cox, J.; Horning, S.; Mann, M.; Makarov, A. Mol. Cell. Proteomics 2012, 11, doi:10.1074/mcp.0111.013698

7. Bateman, K.P., Kellmann, M., Muenster, H., Papp, R., Taylor, L. 2009. J. Am. Soc. Mass Spectrom. 20, 1441-50.

8. Michalski, A.; Damoc, E.; Hauschild, J. P.; Lange, O.; Wieghaus, A.; Makarov, A.; Nagaraj, N.; Cox, J.; Mann, M.; Horning, S. Mol. Cell. Proteomics, 2011, 10, M111.011015.

9. Scheltema, R. A.; Hauschild, J.-P.; Lange, O.; Hornburg, D.; Denisov, E.; Damoc, E.; Kuehn, A.; Makarov, A.; Mann, M. The Q Exactive HF, a Benchtop mass spectrometer with a pre-filter, high performance quadrupole and an ultra-high-field Orbitrap analyzer. Mol. Cell. Proteomics, 2014, 13 (12), 3698-3708.

10. Kelstrup, C.; Bekker-Jensen, D.; Arrey, T.; Hogrebe, A.; Harder, A.; Olsen, J. Performance evaluation of the Q Exactive HF-X for shotgun proteomics. J Proteome Res. 2018, 17, 727-738. 
11. Senko, M., Remes P., Canterbury J., Mathur R., Song Q., Eliuk S., Mullen C., Earley L., Hardman M., Blethrow J., Bui H., Specht A., Lange O., Denisov E., Makarov A., Horning S., Zabrouskov V. Anal Chem. 2013; 85(24):11710-4.

12. Bekker-Jensen, D.B., Martínez-Val, A., Steigerwald, S., Ruether, P., Fort, K.L., Arrey, T.N., Harder, A., Makarov, A.A., Olsen, J.V. Mol. Cell. Proteomics 2020, MCP.TIR119.001906

13. Pfammatter, S., Bonneil, E., McManus, F. P., Prasad, S., Bailey, D. J., Belford, M., Dunyach, J.J., Thibault, P. Mol. Cell. Proteomics 2018, 17(10), 2051-2067.

14. Pino, L. K., Just, S. C., MacCoss, M. J., \& Searle, B. C.Mol. Cell. Prot. (2020) P119.001913.

15. Searle, B. C., Swearingen, K. E., Barnes, C. A., Schmidt, T., Gessulat, S., Küster, B., Wilhelm, M. Nature Communications 2020 11(1), 1-10. 
FIGURES:

Figure 1. Layout of the Orbitrap Exploris quadrupole-Orbitrap family of mass spectrometers:

a) Outside view and modular concept,

b) Ion-optical path of the instrument with its major elements labeled.

Figure 2. Box plot graph of mass stability over prolonged periods of time as measured on Orbitrap Exploris $120 \mathrm{MS}$ :

a) With external calibration at the start of Day 1 ,

b) With internal calibration using an EASY-IC source to produce an in-spectrum lock-mass.

Daily interquartile ranges (IQR) for 16 selected VetDrug compounds are indicated by boxes, whiskers correspond to 1.5 IQR, points- to outliers. Red bands indicate $\pm 500 \mathrm{ppb}$ mass deviation.

Figure 3. Isotope pattern of biotin $\left(\mathrm{C}_{10} \mathrm{H}_{16} \mathrm{~N}_{2} \mathrm{O}_{3} \mathrm{~S}\right)$ measured in negative mode at different Orbitrap resolution settings (30,000 to 240,000). Fine isotopic structure is not resolved for A1 and A2 isotopes at resolution settings of 30,000 and 60,000 and is resolved with resolution setting of 120,000 and greater, allowing unambiguous determination of the elemental composition of the analyte.

Figure 4. Mass stability during scan-to-scan polarity switching on the example of mycotoxin Zearalenone with external mass calibration on the Orbitrap Exploris 120 MS:

a) chromatographic trace, with black sticks indicating acquisition in positive polarity and red sticks- in negative polarity,

b) mass deviation from accurate mass of the precursor in positive polarity,

c) mass deviation from accurate mass of the precursor in negative polarity.

Figure 5. Pesticide analysis in olive oil for $>500$ pesticides using Orbitrap Exploris 240 MS:

a) chromatographic trace of pesticides with different analytes indicated by different colors. Insert enlarges the densest part of the chromatogram, indicating a sufficient number of scans across each elution profile to provide good quantitation.

b) achieved limits of quantitation (LOQs) and detection (LODs) for analyzed pesticides.

Figure 6. Comparison of MS/MS coverage of human plasma (NIST SRM1950) on the Orbitrap Exploris 240 MS using a) traditional DDA approach and b) AcquireX workflow. AcquireX intelligent acquisition significantly increased the number of compounds with fragmentation data in human plasma over traditional DDA after three injections. The circle represents the total number of 
compounds detected in human plasma and the share of compounds without corresponding MS/MS spectra is shown in pink.

Figure 7. Spectra of intact trastuzumab monoclonal antibody acquired at a resolution setting of 30,000:

a) under native conditions using SEC LC/MS,

b) under denaturing conditions using RP LC/MS,

c) comparison of intact mass distributions under native (top) vs. denaturing conditions (bottom) after deconvolution, with mass errors in green.

Figure 8. RP LC/MS analysis of trastuzumab following digestion using Fabricator (IdeS) and disulfide reduction:

a) total ion chromatograms of separated subunits,

b) deconvolution of monoisotopic masses from obtained full scan mass spectra,

c) panoramic spectra and baseline resolved isotope patterns of the individual charge states of subunits.

Figure 9. Performance of the Orbitrap Exploris 240 and Orbitrap Exploris 480 MS in bottom-up DDAFAIMS proteomic experiments at different sample loads:

a) the average number of identified unique protein groups,

b) the average number of identified unique peptides.

Arrows and per cent values indicate the improvement in identifications from Orbitrap Exploris 240 to $480 \mathrm{MS}$. Two FAIMS compensation voltages (-50V and $-70 \mathrm{~V})$ with intra-analysis switching were used for both instruments.

Figure 10. Performance of the Orbitrap Exploris $240 \mathrm{MS}$ in a bottom-up DIA proteomic experiment for different proportions of $E$. Coli spiked in a Pierce HeLa tryptic digest sample ( $0 \%$ corresponding to pure HeLa analysis). Total sample load was $1 \mu \mathrm{g}$ in all experiments. The number of proteins in the library is indicated by a blue dashed line. 
Figure 1a)
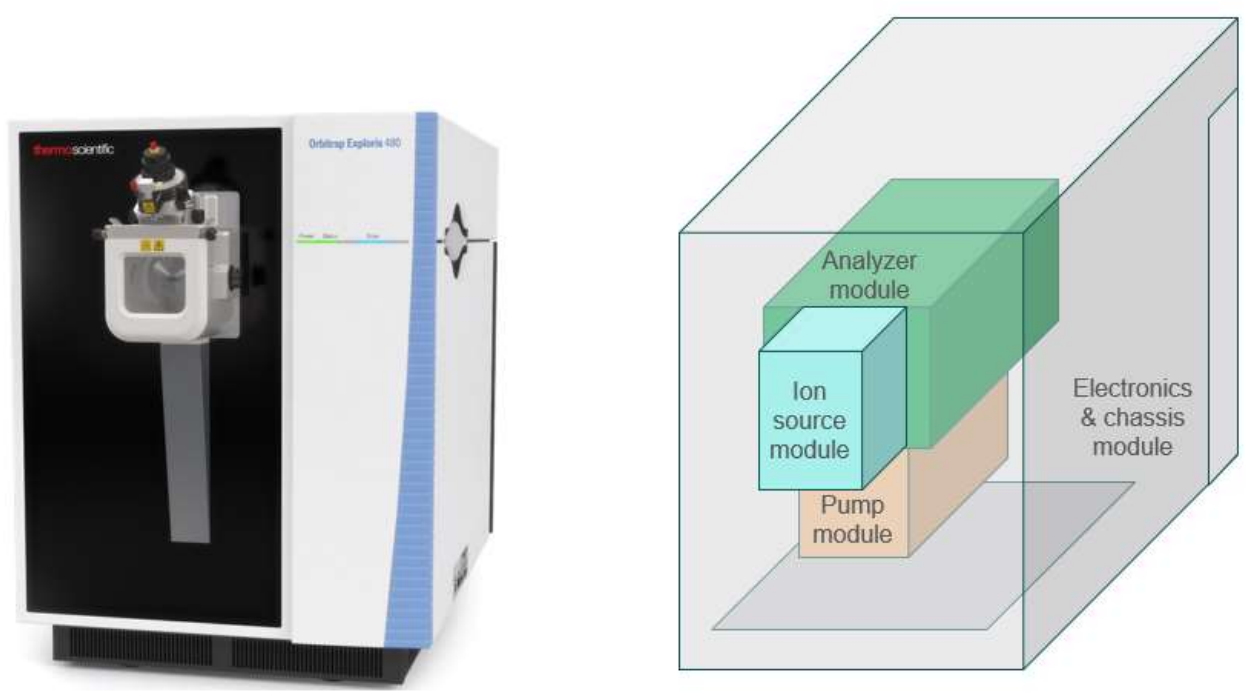

Figure 1b)

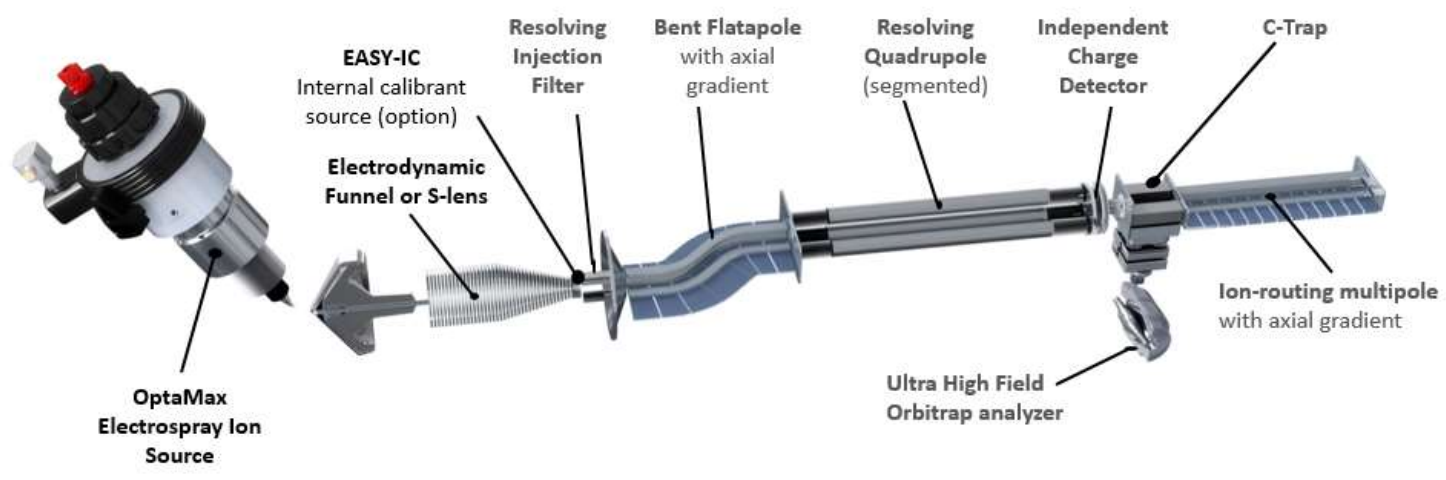


Fig. 2. Mass stability

a)

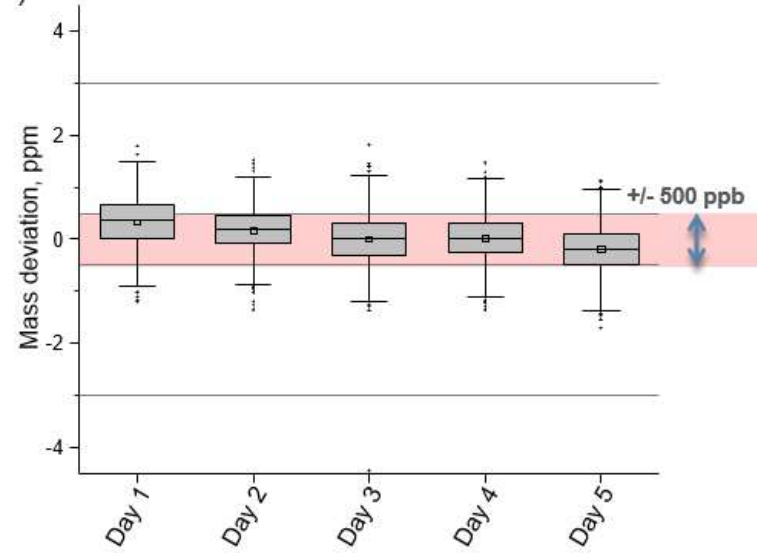

b)

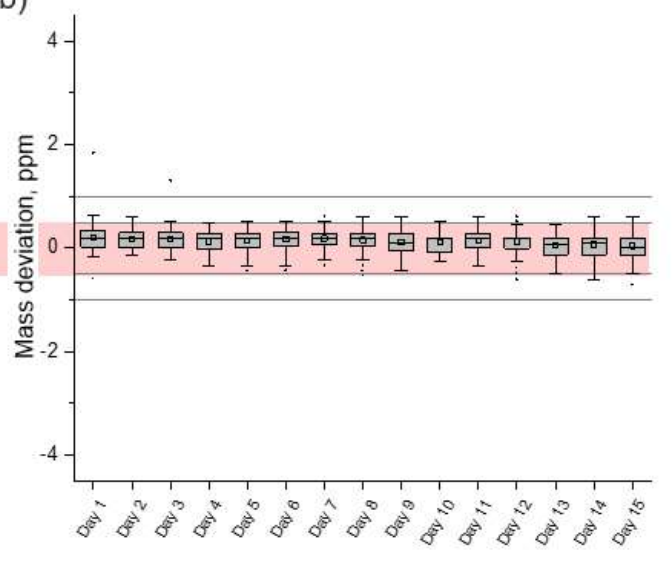

Fig. 3.
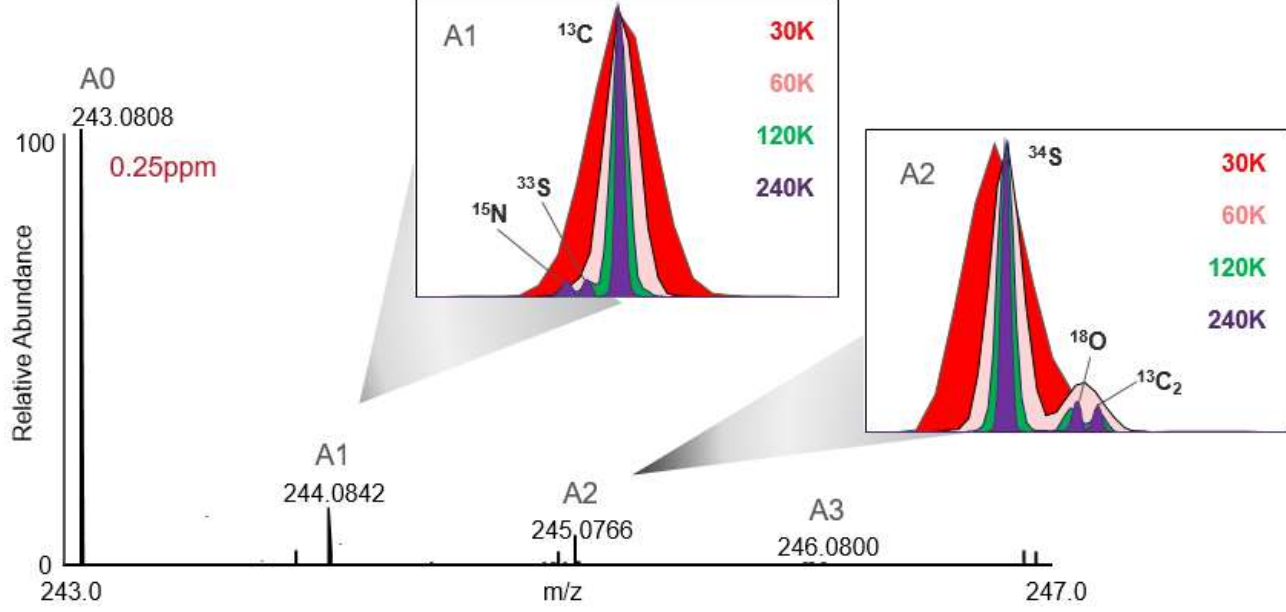

A3

246.0800

1 
Figure 4.

a)

b)

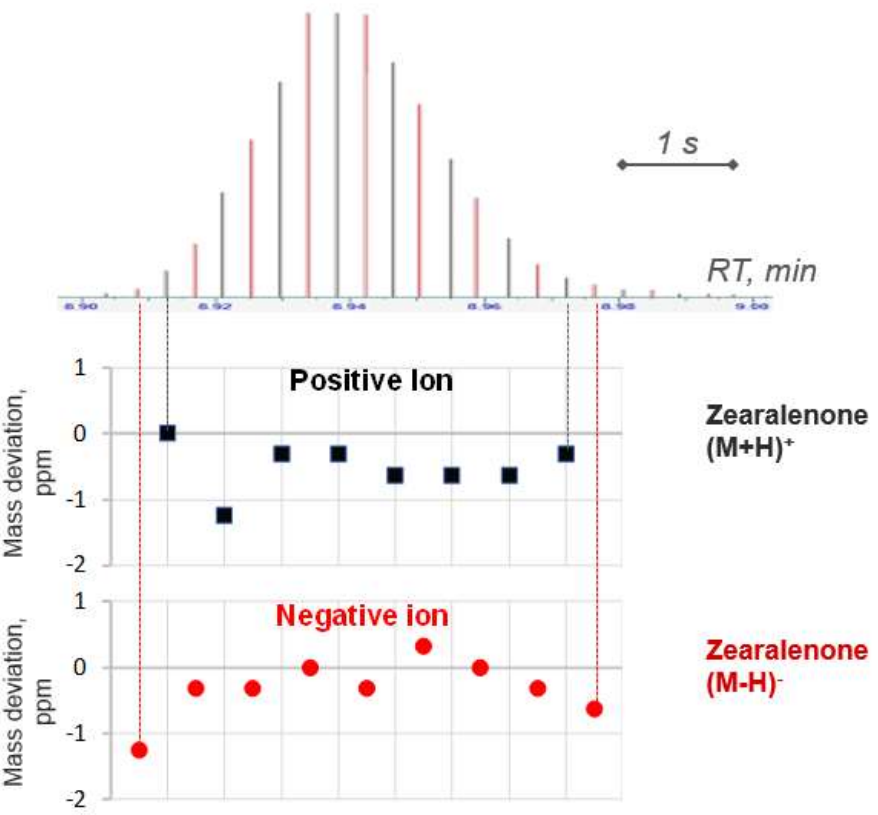

Figure 5.

a)

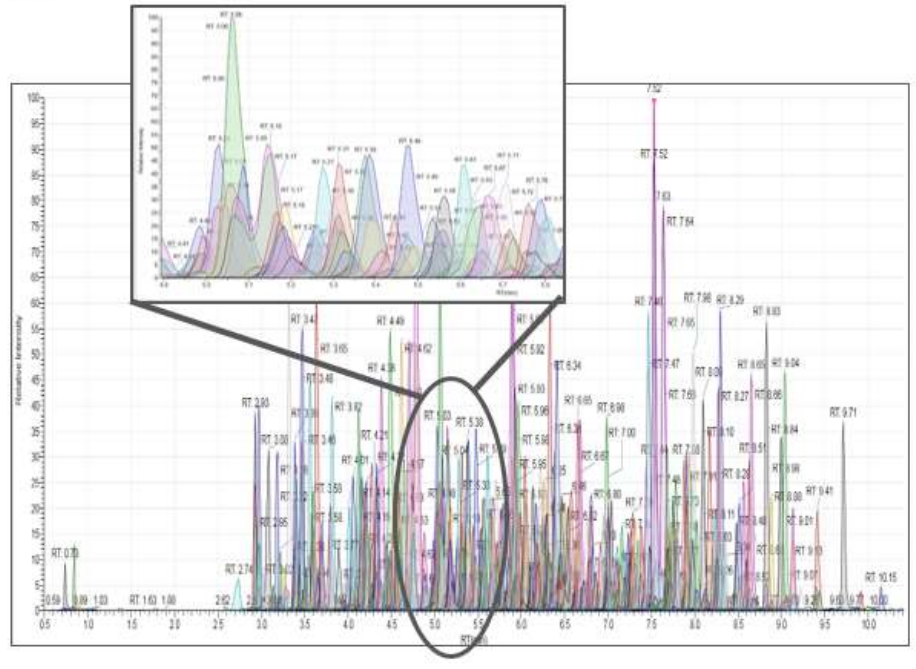

b)

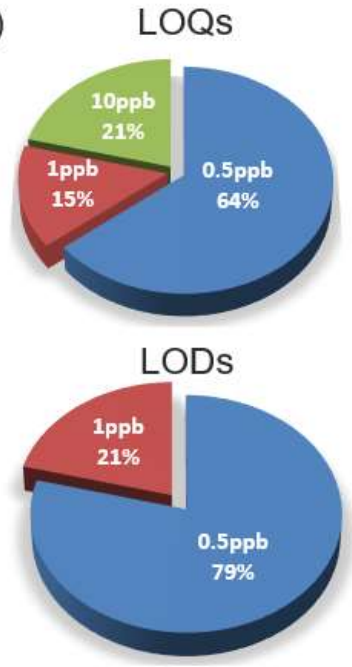


Figure 6.

a)
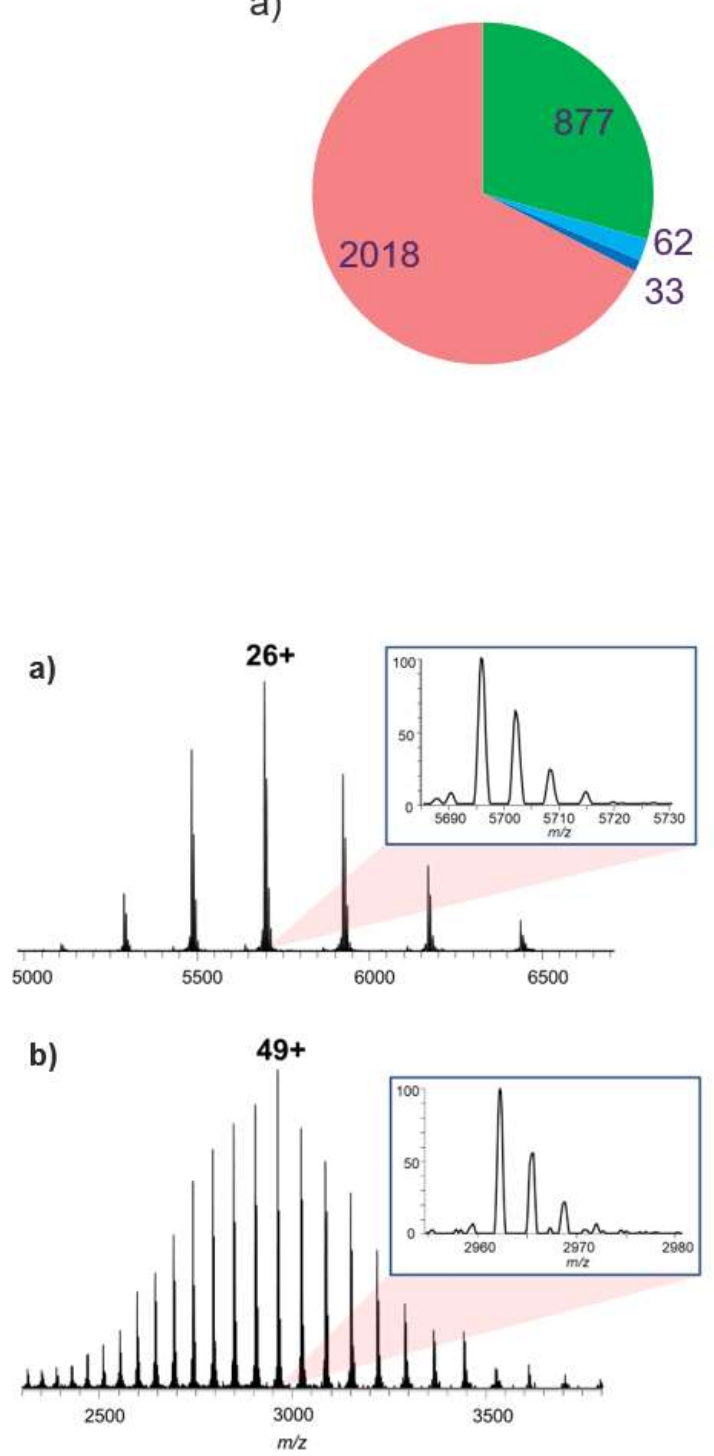

b)
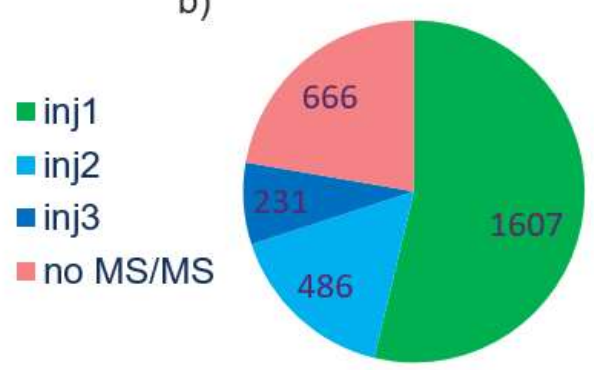

c)

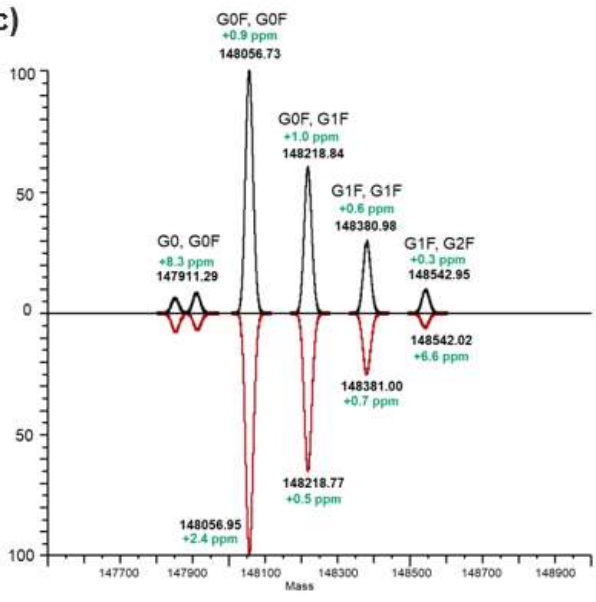

Fig. 7 
Fig. 8

a)

c)
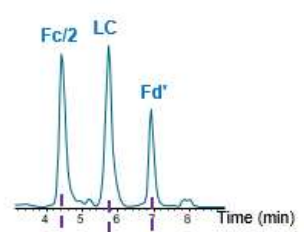

)

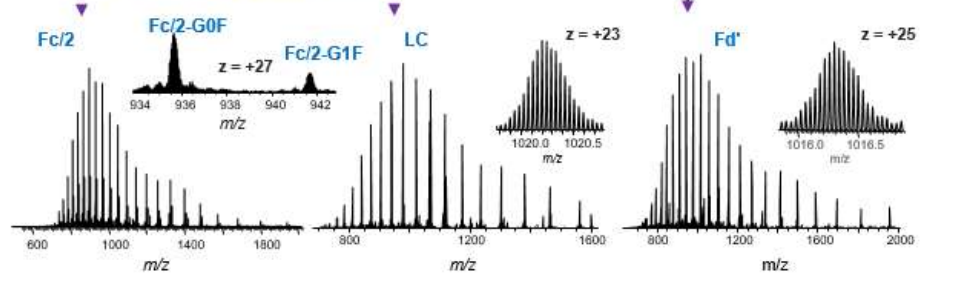

b) $\quad \mathrm{LC}+2.0 \mathrm{ppm}$

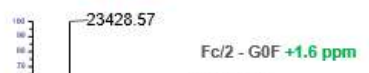

25220.50

$25367.55 \quad \mathrm{Fd}^{\prime}+1.1 \mathrm{ppm}$

$25382.53 \quad \mathrm{Fc} / 2-\mathrm{G} 1 \mathrm{~F}+0.7 \mathrm{ppm}$

Figure 9.

a)

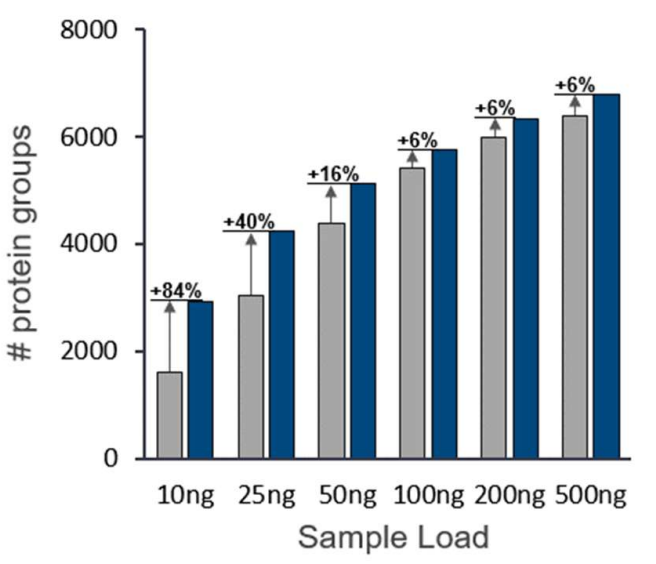

Orbitrap Exploris 240 MS b)

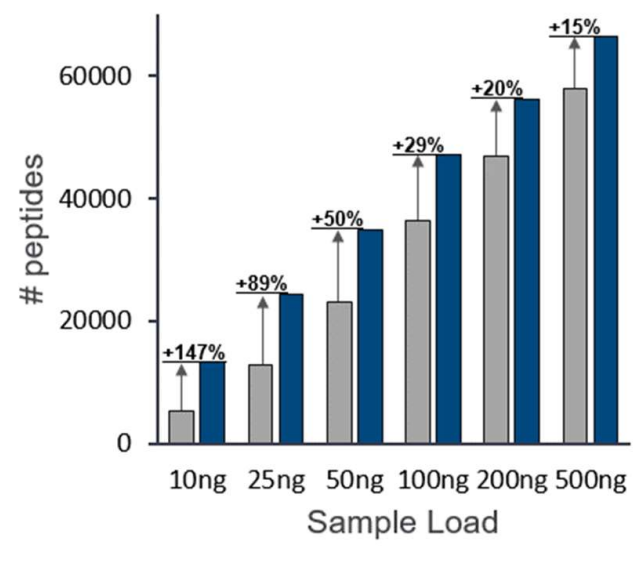

Orbitrap Exploris 480 MS 
Figure 10.

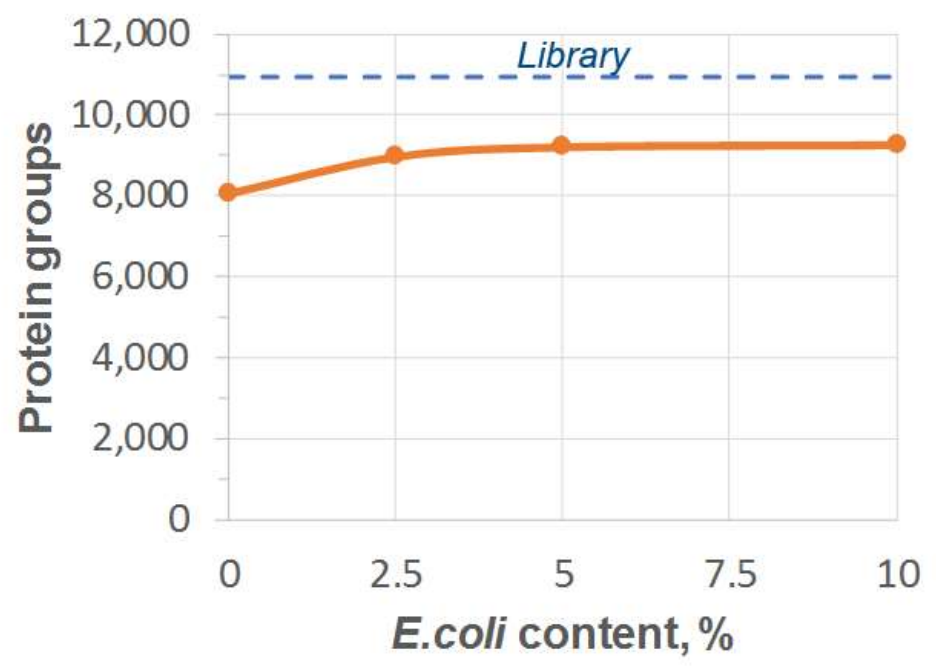




\section{SUPPLEMENTARY INFORMATION}

\section{A Novel Family of Quadrupole-Orbitrap Mass Spectrometers for a Broad Range of Analytical Applications}

Jan-Peter Hauschild ${ }^{1}$, Amelia Peterson ${ }^{1}$, Erik Couzijn ${ }^{1}$, Eduard Denisov ${ }^{1}$, Denis Chernyshev ${ }^{1}$, Christian Hock ${ }^{1}$, Hamish Stewart ${ }^{1}$, Ralf Hartmer ${ }^{1}$, Dmitry Grinfeld ${ }^{1}$, Christian Thoeing ${ }^{1}$, Bastian Reitemeier ${ }^{1}$, Arne Kreutzmann'1, Oliver Lange ${ }^{1}$, Wilko Balschun ${ }^{1}$, Aivaras Venckus ${ }^{1}$, Sebastian Kanngiesser ${ }^{1}$, Alexander Kholomeev ${ }^{1}$, Gregor Quiring ${ }^{1}$, Frank Czemper ${ }^{1}$, Eloy Wouters ${ }^{2}$, Michael Belford $^{2}$, Mathias Mueller ${ }^{1}$, Jens Grote ${ }^{1}$, Tabiwang N Arrey ${ }^{1}$, Julia Kraegenbring ${ }^{1}$, Kerstin Strupat ${ }^{1}$, Markus Kellmann ${ }^{1}$, Siegrun Mohring ${ }^{1}$, Catharina Crone ${ }^{1}$, Alexander Harder ${ }^{1}$, Aaron M. Robitaille ${ }^{2}$, Khatereh Motamedchaboki², Yang Liu², Aaron S. Gajadhar², Daniel Lopez Ferrer², Eugen Damoc ${ }^{1}$, Angela Criscuolo ${ }^{1}$, Kristina Srzentic ${ }^{2}$, Ed George ${ }^{2}$, Charles Yang ${ }^{2}$, Ioanna Ntai², Amanda Souza ${ }^{2}$, Andreas Wieghaus ${ }^{1}$, Alexander Makarov ${ }^{1 *}$

1- Thermo Fisher Scientific, Bremen, Germany

2- Thermo Fisher Scientific, San Jose, CA, USA 


\section{Section 1. Detailed Description of the quadrupole/Orbitrap instrument.}

\section{Design and operation of the instrument}

Ions formed at atmospheric pressure (in this work, in an electrospray ion source, Figure 1b) pass through the heated transfer tube into the atmosphere-to-vacuum interface with an RF-powered S-lens or electrodynamic funnel and then through a mass-selective injection filter operating at switchable RF frequencies of $\sim 500$ or $\sim 1000 \mathrm{kHz}$ up to $600 \mathrm{~V} \mathrm{p}-\mathrm{p}$ as described in [1] (please see references after the Section 2). Further details of the interface for each model of the instrument family are presented below.

Between the S-lens or funnel and the injection filter, the EASY-IC discharge ion source [2] provides a stable flow of fluoranthene ions as lock masses at $\mathrm{m} / \mathrm{z} 202.0777$ and $\mathrm{m} / \mathrm{z} 202.0788$ for positive and negative polarity, respectively, thus enabling self-diagnostics and ppm-level mass stability over many days.

Following optional rough mass filtering by the injection filter, the ions enter a bent flatapole through an accelerating lens aperture (detailed schematics of each variant are shown in Figure S-1). The Sshaped quadrupolar bent flatapole operates at $2.5 \mathrm{MHz}$ and up to $1000 \mathrm{~V} \mathrm{p}-\mathrm{p} \mathrm{RF}$. It consists of a pair of parallel PCBs with flat metal rods with $4 \mathrm{~mm}$ separation between rod faces. The rods are oriented in such a way that clusters and droplets are directed along the line-of-sight from the heated transfer tube between the flatapole rods onto a dump. The resulting neutral particles are efficiently pumped out of the module through openings in the parallel PCBs near the dump. These openings have been adjusted to balance the density of background gas inside the bent flatapole for appropriate collisional cooling of the ions at a minimum extent of clustering, e.g., with solvent molecules. The ions are driven through the bent flatapole by an axial electric field created by a voltage divider array on PCB stripes similar to that described in [1]. After collisional cooling in the bent flatapole, the ions are transmitted via an accelerating lens aperture of $2.5 \mathrm{~mm} I D$ into a hyperbolic segmented-rod quadrupole $(\mathrm{r} 0=4$ $\mathrm{mm}$, length $170 \mathrm{~mm}$ ) operated at $860 \mathrm{kHz}$.

The known issue of excessive contamination of one rod pair of a quadrupole mass filter under heavy ion load with continuous operation has been addressed by introducing symmetrical ion loading in Orbitrap Exploris 240 and Orbitrap Exploris 480 MS. In this mode, contamination is symmetrically applied to the rods by automatically and regularly switching the resolving DC polarity of the quadrupole rod pairs, thereby changing the distribution of filtered-out ions that get deposited onto the rods. The quadrupole endurance score, first introduced in $Q$ Exactive instruments, reflects the drift of isolation shape as a function of ion load on the instrument, with a score of 1.0 representing a clean quadrupole. Longitudinal studies with infusion of a concentrated solution of ubiquitin demonstrate that this score degrades slower when using symmetrical ion loading compared to traditional single rod-pair operation (Figure S-2). The introduction of symmetrical ion loading of the quadrupole required improvements to quadrupole manufacturing tolerances so that both rod polarities provide equivalent transmission and isolation quality. 
As ions leave the quadrupole mass filter, they get modulated by a split lens now integrated with an independent charge detector. Besides either transmitting or blocking the ion beam, this split lens has a third, intermediate mode in which the ion beam is deflected onto an electrometer surface. The electrometer reading is used for occasional correction of the automatic gain control (AGC) algorithm run on the Orbitrap analyzer. In the transmission mode, ions fly through a short transport multipole via the C-trap into a nitrogen-filled lon Routing Multipole (IRM) flatapole collision cell, where they are trapped and, if requested, fragmented. A single RF supply with ferrite coils drives the C-trap, transport multipole, and IRM at 3.2 MHz frequency.

The IRM device consists of a pair of parallel PCBs with straight, flat metal rods with 4 mm separation between rod surfaces; a voltage divider array on PCB stripes provides an adjustable axial field gradient. The flatapole is further enclosed by two metal side walls that provide precise spacing of the parallel PCBs, a back-end PCB electrode, and a flat metal entrance lens interfacing it to the C-trap. The IRM is filled with ultra-high purity nitrogen from a gas line. The pressure in the cell is measured directly by a Pirani gauge and can be regulated in the range from $3^{*} 10^{-3}$ to $18^{*} 10^{-3}$ mbar in the intact protein mode. Like in all other Orbitrap instruments of the $Q$ Exactive [1, 3-4] and Orbitrap Fusion series [2], higher energy collisional dissociation (HCD) of ions in the IRM is achieved by adjusting the DC offset of the RF rods to provide the required collision energy, with the axial field gradient defining the resting location of ions prior to their return to the C-trap. Multiple precursor ions can be fragmented sequentially at their optimum collision energies without compromising the storage of preceding injections.

The return transfer of ions from the IRM into the C-trap was optimized for high ion loads and throughput. Specifically, a pre-purge pulse is applied, lowering the DC offset of the IRM rods to move away any ions residing close to the IRM entrance lens, so that they will experience a proper focusing field during the ensuing purge event. Once rapid elevation of the IRM offset and axial field initiates ion transfer, the IRM offset, the axial field, and the C-trap lens voltages continue to be slowly ramped up during the transfer so that the ions are subjected to an increasing potential barrier as they approach lenses during reflections within the $\mathrm{C}$-trap and therefore cannot get deposited onto lens edges. Figure S-3 demonstrates the resulting reduction of contamination of the C-trap lenses under heavy ion load.

Once ions have been cooled inside the C-trap by the gas leaking in from the IRM, voltages on all its electrodes are raised to the optimum level for injection into the Orbitrap analyzer. Unlike all previous C-trap designs, the same extraction voltage is applied to all electrodes of the trap except the slotted pull-out electrode. This ensures strong ion focusing upon extraction and reduces losses on the edges of the slot (Figure S-4), although such an approach imposes the need for an additional focusing lens on the way to the Orbitrap analyzer.

As the result of these provisions, stable operation of the mass spectrometer over long periods of time was demonstrated even for the most sensitive and therefore the most susceptible to contamination Orbitrap Exploris 480 instrument (Figure S-5). As the Orbitrap Exploris 120 and Orbitrap Exploris 240 instruments transmit 3-5-fold fewer ions through ion optics, the interval between cleanings is expected to be longer for these models by about the same factor. 
The horizontally aligned and symmetrically supported ultra-high-field Orbitrap analyzer is open to pumping on both ends and combines improved mechanical and electrical balancing with better vacuum inside the trap, enabling higher-resolution analysis with longer transients compared to previous platforms. Ions are detected with $4 \mathrm{kV}$ applied to the trap, which is somewhat lower than the $5 \mathrm{kV}$ applied in previous instruments to allow a more compact design.

Unlike previous Orbitrap instrument families, pulsing of the central electrode has an adjustable starting point in time prior to the pull-out pulse on the C-trap. With an adjustable starting voltage and a fixed ramp time constant determined by the Orbitrap capacitance and an input resistor, this enables electrodynamic squeezing in the trap [5] of a very broad mass range: $\mathrm{m} / \mathrm{z} 40$ to 8000 . This feature additionally allows use of a single calibration voltage set for the entire mass range.

\section{Processing of Transients}

Transient image currents for the ion packets that move forth and back in the Orbitrap mass analyzer are amplified by a pre-amplifier, then filtered and digitized by a data acquisition board plugged into the base-plane board. During filtering, spectral noise peaks at the frequencies of the quadrupole mass filter and other RF devices are actively cancelled by mixing in a pick-up from the RF devices by a previously calibrated proportion.

The resulting digital waveform is then processed by a built-in PC using an enhanced version of Fourier Transformation (eFT ${ }^{\mathrm{TM}}$ ) as described in [6]. For Tandem Mass Tag $^{\text {TM }}$ (TMT ${ }^{\mathrm{TM}}$ ) experiments, narrow $\mathrm{m} / \mathrm{z}$ regions near TMT-10 reporter peaks $[7,8]$ are processed by the Phase-constrained signal deconvolution method (Fi-Transform) as described in [9]. Fi-Transform allows an effective doubling of the mass resolution and use of transient durations of 32 and $64 \mathrm{~ms}$ (resolution settings 15,000 and 30,000 at $\mathrm{m} / \mathrm{z} 200$ ), increasing the scan rate of acquisition for TMT experiments while maintaining sufficient resolution to baseline resolve TMT isotopologues (so-called TurboTMT mode).

\section{Instrument control software}

The instrument control software (ICSW) links together all boards of the instrument and runs on an onboard industrial PC. It is organized on multiple levels: all procedures for the control of ion optics are written in Python, while signal processing is performed by $\mathrm{C}++$ programs. The built-in PC communicates over a gigabit ethernet with an external Application PC that supports the graphical user interface including saving and processing of RAW files. USB, ethernet and general purpose I/O interfaces are available for communication with LC and other external devices.

The Orbitrap Exploris ICSW was engineered to provide a similar look-and-feel to the corresponding ICSW of the Tribrid and TSQ platforms. Many settings, such as absolute collision energy (CE) and normalized collision energies (NCE) of higher-energy collisional dissociation (HCD) fragmentation, were harmonized between these different instruments. This software also enables automatic bakeout of the system after venting and one-click calibration with pause and restart options once the instrument is ready for operation.

For improved serviceability, the Orbitrap Exploris family permits customer cleaning not only of the front-end ion optics, but also of the analytical quadrupole using a dedicated cleaning kit. All 
instruments contain also provisions for remote system monitoring by the service organization as well as by users using Thermo Scientific ${ }^{\mathrm{TM}}$ Almanac web-based application.

\section{Orbitrap Exploris 120, 240 and 480: Instrument configurations.}

This work presents three different high-resolution accurate mass LC/MS instrument models based on the new design that collectively address a broad range of important analytical applications.

The Orbitrap Exploris $120 \mathrm{MS}$ is designed for highest performance in quantitative environmental, food safety and toxicology analysis, as well as other small-molecule applications. It provides resolving powers from 15,000 to 120,000 at m/z 200 (32 to 256 ms transients, respectively) and has a white panel on the front instead of a black one of Figure 1a. It utilizes a round-bore transfer tube with 580 $\mu \mathrm{m} I D$ followed by an S-lens operated at $650 \mathrm{kHz}$. Its pumping system consists of a six-stage TurboVac Hexalnlet TMP and a single-stage rotary vane pump Sogevac SV65BI (both from Leybold $\mathrm{GmbH}$, Cologne, Germany). Its IRM pressure is fixed at a standard setting of $1.1 \cdot 10^{-2} \mathrm{mbar}$.

The Orbitrap Exploris $240 \mathrm{MS}$ is designed as a universal instrument that could also address -omics, pharmaceutical and bio-pharmaceutical applications. It provides resolving powers from 15,000 to 240,000 at $\mathrm{m} / \mathrm{z} 200$ (32 to $512 \mathrm{~ms}$ transients, respectively) and features a silver front panel on Figure 1a. It also utilizes a round-bore transfer tube with $580 \mu \mathrm{m}$ ID followed by an S-lens operated at 650 kHz. Its pumping system consists of a 6-stage SplitFlow XL TMP (Pfeiffer Vacuum, Asslar, Germany) and a rotary pump Sogevac SV65. Alternatively, both Orbitrap Exploris 120 and 240 MS could be backed by an optional Leybold Ecodry 65 Plus multi-stage roots vacuum pump that offers significantly decreased noise level, size and power consumption.

The Orbitrap Exploris 480 MS was introduced in [10] as the instrument optimized for -omics and biopharmaceutical applications. It features resolving powers from 7,500 to 480,000 at m/z 200 (16 to $1024 \mathrm{~ms}$ transients, respectively) and has a black front panel as shown on Figure 1a. It features also TurboTMT mode. The mass spectrometer utilizes a HCTT of $0.6 \times 1.6 \mathrm{~mm}$ cross-section followed by an electrodynamic funnel operated at $1800 \mathrm{kHz}$. Its pumping system consists of a six-stage SplitFlow XL TMP (Pfeiffer Vacuum, Asslar, Germany) and two rotary vane pumps: a Sogevac SV120BI (Leybold $\mathrm{GmbH}$, Cologne, Germany) for pumping of the funnel region and a Pfeiffer DUO11 (Pfeiffer Vacuum, Asslar, Germany) for backing of the TMP. Alternatively, the mass spectrometer could be backed by an optional purpose-developed Edwards nXL110iD dual-inlet dry pump (Edwards GmbH, Feldkirchen, Germany) with significantly decreased noise level, size and power consumption.

For both the Orbitrap Exploris 240 and 480 MS, a so-called "Biopharma" option allows operation of the instrument at different IRM pressure modes to provide optimal conditions for two application scenarios. First, to obtain isotopically resolved spectra of small- and medium-size intact proteins, the pressure is lowered to achieve the best possible vacuum inside the Orbitrap analyzer. Second, for the analysis of larger molecules like antibodies under native conditions up to $\mathrm{m} / \mathrm{z} 8000$, the pressure in the IRM can be raised to optimize the trapping and sensitivity of these compounds.

Also, both the Orbitrap Exploris 240 and 480 MS can be equipped with the optional FAIMS Pro interface to enable atmospheric-pressure separation of ions based on non-linear mobility in the field- 
asymmetric waveform ion mobility spectrometer (FAIMS). Details of the design and characterization of the FAIMS Pro interface are presented in [11]. A unique feature of this device is the use of cylindrical electrodes that help focus ions through the electrode assembly as they are transported by the nitrogen carrier gas from inlet to outlet. With the inner electrode blocking the "line-of-sight" for clusters and dust particles, the interface provides an additional benefit of protection from environmental factors. As shown in [10], the addition of the FAIMS filtering enables deeper analysis of the proteome even with short LC gradients, and significantly increases the number of identified proteins, typically by $15-$ $20 \%$.

Lastly, all instruments of the new family contain a shelf under a foldable cover on the right side, where the syringe pump (Chemyx SKE10, Stafford, TX, USA) and an optional 6-port inject valve MX Series II ${ }^{\mathrm{TM}}$ (IDEX Health \& Science LLC, Rohnert Park, CA) can be placed in close proximity to the ion source for infusion or loop injection of calibration mixtures or samples.

\section{Section 2. Experimental procedures for experiments on ion optics contamination.}

Contamination experiments with quadrupole (Figure S-2) and C-trap ion optics (Figure S-3, S-4) employed a $2.9 \mathrm{pmol} / \mu \mathrm{L}$ concentration of ubiquitin from bovine erythrocytes (Sigma Aldrich, St. Louis MO, USA) in a $50 \%$ acetonitrile, $0.1 \%$ formic acid solution in water. For direct infusion of the ubiquitin solution, a Thermo Scientific ${ }^{\text {TM }}$ Accela ${ }^{\text {TM }} 600$ pump (Thermo Fisher Scientific, Austin, TX, USA) was configured for isocratic flow at $10 \mu \mathrm{L} / \mathrm{min}$.

For quadrupole contamination experiments of Figure S-2, an instrument-controlled divert valve automatically infused FlexMix calibration solution via a syringe pump for approximately $1 \mathrm{hr}$ at 10 $\mu \mathrm{L} / \mathrm{min}$ every $8 \mathrm{hr}$ to assess quadrupole performance. During ubiquitin measurement, the system was configured to run a Top10 DDA experiment on the most abundant charge states of ubiquitin with maximum ion load (50 ms fixed injection times). Data analysis was carried out via a Jupyter Notebook using Python 3.6.

For C-trap exit lens contamination experiments in Figure S-3, the instrument was operated under prolonged (up to $97 \mathrm{hr}$ ) infusion of the same ubiquitin solution to mimic a high ion flux from ion source which is expected to induce contamination. During the exposure, the instrument was operated at 50 $\mathrm{Hz}$ without quadrupole isolation and a target $\mathrm{m} / \mathrm{z}$ range of 150-2000 to maximize ion load.

Contamination effects were measured by regularly scanning the C-trap Exit Lens voltage during the purge to C-trap and monitoring the signal intensity of ions. Meanwhile, all ubiquitin ions were fragmented at $35 \mathrm{~V}$ normalized collision energy to produce a wide range of $\mathrm{m} / \mathrm{z}$ ions to monitor from $\mathrm{m} / \mathrm{z} 40$ to 1400 . This mass range corresponds to a very low C-trap RF amplitude ( $150 \mathrm{~V} p-\mathrm{p}$ ) and very poor trapping of the higher $\mathrm{m} / \mathrm{z}$ ions in order to greatly exacerbate any negative effects of aperture charging. This extreme treatment was necessary to observe relatively small charging effects over short experimental cycles. The overall signal and background pressures were monitored to ensure consistent exposure rates, and lenses were cleaned between experimental runs. A scheme for 
dynamic ramping of the IRM and Exit Lens was designed to provide a $0.8 \mathrm{~V} / \mathrm{ms}$ ramp for $3 \mathrm{~ms}$.

Experimental runs were carried out with and without the dynamic ramping process applied with results shown in Figure S-3b.

For characterization of the most sensitive instrument under real-life sample load, two Orbitrap Exploris 480 mass spectrometers were run with Thermo Scientific ${ }^{\text {TM }}$ UltiMate $^{\text {TM }} 3000$ RSLC Nano UHPLC with Easy-Spray 806A column. To imitate the harshest conditions, the system ran DDA experiments of human plasma samples (provided by Pierce, Rockford, IL). As represented by Figure S-5b, three QC runs were inserted after each 10 plasma injections, each using Pierce HeLa spiked with Thermo Scientific ${ }^{\mathrm{TM}}$ Pierce $^{\mathrm{TM}}$ Peptide Retention Time Calibration Mixture (PRTC). Protein identification performance for non-stop operation over many months is presented on Figure S-5b. 


\section{References}

1. Michalski, A.; Damoc, E.; Hauschild, J. P.; Lange, O.; Wieghaus, A.; Makarov, A.; Nagaraj, N.; Cox, J.; Mann, M.; Horning, S. Mol. Cell. Proteomics, 2011, 10, M111.011015.

2. Senko, M., Remes P., Canterbury J., Mathur R., Song Q., Eliuk S., Mullen C., Earley L., Hardman M., Blethrow J., Bui H., Specht A., Lange O., Denisov E., Makarov A., Horning S., Zabrouskov V. Anal Chem. 2013; 85(24):11710-4.

3. Scheltema, R. A.; Hauschild, J.-P.; Lange, O.; Hornburg, D.; Denisov, E.; Damoc, E.; Kuehn, A.; Makarov, A.; Mann, M. The Q Exactive HF, a Benchtop mass spectrometer with a pre-filter, high performance quadrupole and an ultra-high-field Orbitrap analyzer. Mol. Cell. Proteomics, 2014, 13 (12), 3698-3708.

4. Kelstrup, C.; Bekker-Jensen, D.; Arrey, T.; Hogrebe, A.; Harder, A.; Olsen, J. Performance evaluation of the Q Exactive HF-X for shotgun proteomics. J Proteome Res. 2018, 17, 727-738.

5. Makarov, A. A. Anal. Chem. 2000, 72, 1156.

6. Lange, O.; Damoc, E.; Wieghaus, A.; Makarov, A. Enhanced Fourier transform for Orbitrap mass spectrometry. Int. J. Mass Spectrom. 2014, 369, 16-22.

7. McAlister, G. C.; Huttlin, E. L.; Haas, W.; Ting, L.; Jedrychowski, M. P.; Rogers, J. C.; Kuhn, K.; Pike, I.; Grothe, R. A.; Blethrow, J. D.; et al. Increasing the multiplexing capacity of TMTs using reporter ion isotopologues with isobaric masses. Anal. Chem. 2012, 84 (17), 7469-7478.

8. Werner, T.; Becher, I.; Sweetman, G.; Doce, C.; Savitski, M. M.; Bantscheff, M. High resolution enabled TMT 8-plexing. Anal. Chem. 2012, 84 (16), 7188-7194.

9. Kelstrup, C.; Aizikov, K.; Batth, T.; Kreutzman, A.; Grinfeld, D.; Lange, O.; Mourad, D.; Makarov, A.; Olsen, J. Limits for Resolving Isobaric Tandem Mass Tag Reporter lons Using PhaseConstrained Spectrum Deconvolution. J Proteome Res. 201817 (11), 4008-4016.

10. Bekker-Jensen, D.B., Martínez-Val, A., Steigerwald, S., Ruether, P., Fort, K.L., Arrey, T.N., Harder, A., Makarov, A.A., Olsen, J.V. Mol. Cell. Proteomics 2020, MCP.TIR119.001906

11. Pfammatter, S., Bonneil, E., McManus, F. P., Prasad, S., Bailey, D. J., Belford, M., Dunyach, J.J., Thibault, P. Mol. Cell. Proteomics 2018, 17(10), 2051-2067. 


\section{SUPPLEMENTARY FIGURES}

Figure S-1. Schematics of the Orbitrap Exploris family of instruments:

a) Orbitrap Exploris 120 and Orbitrap Exploris 240 MS,

b) Orbitrap Exploris $480 \mathrm{MS}$.

Figure S-2. Quadrupole endurance score as a function of sample load on the instrument for symmetrical (green line and points) and traditional, unidirectional ion loading (blue line and points) of the quadrupole mass filter. The quadrupole endurance score reflects the changes and shifts in isolation shape as measured by a loss in isolation transmission that occurs with material deposition on the quadrupole. A score of 1.0 represents a clean, maximally transmissive quadrupole. The data in this figure span over multiple clogging and cleaning cycles of the transfer tube while the instrument was contaminated by infusion of a concentrated solution of ubiquitin. Wide bands around lines indicate the corresponding r.m.s. spread of the score and reflect the resulting changes over time.

At around $3.3 \mathrm{mg}$ of ubiquitin (resulting from $221 \mathrm{hr}$ of continuous infusion of a $2.9 \mathrm{pmol} / \mu \mathrm{l}$ ubiquitin solution at $10 \mu \mathrm{l} / \mathrm{min}$ ), the drop of performance reaches a critical threshold for the traditional method, but for the symmetrical ion loading method, high performance is maintained and usable analyses could continue for several times more sample load. Further experimental details are presented in Section 2.

Figure S-3. Ion transfer to the C-trap.

a) For ion return from the IRM to the C-trap, the IRM offset, the axial field gradient, and the Ctrap lens voltages are slowly ramped up so that ions are prevented from landing on lens edges.

b) Evolution of tuning curve of the C-trap exit lens voltage with increasing sample load for a ubiquitin fragment ion at $\mathrm{m} / \mathrm{z} 817.3$ without voltage ramp (above) and with the ramp (below) over $>80 \mathrm{hr}(>1.2 \mathrm{mg}$ ) of sample load. The voltage ramp both decreases change on the right side of the plot and broadens the acceptance range on the left side.

Further experimental details are presented in Section 2.

Figure S-4. Ion ejection from the C-trap.

Once ions have been collisionally cooled inside the C-trap, they are extracted by an extraction pulse. The same extraction voltage is applied to all electrodes of the trap, except the pull-out electrode containing an ion-exit slit, to ensure strong ion focusing upon extraction so that losses on the edges of the slot are also reduced. While strong focusing reduces losses and contamination on the exit slit, such extraction requires an additional focusing lens on the way to the Orbitrap analyzer. 
Figure S-5. Experiments to determine interval between cleanings of the Orbitrap Exploris 480 MS.

a) Scheme of the experiment: ten injections of $1 \mu \mathrm{g}$ of plasma is followed by 3 quality control tests, with continuously running cycles of $16 \mathrm{~h}$ and approximately $17 \mu \mathrm{g} / \mathrm{day}$ load.

b) Time series of daily averages of identified protein groups, normalized to the number at the starting time point. Two different instruments were used for the experiment, with instrument 1 undergoing full cleaning and update of software between study 1 and 2. Instrument 2 (3rd study) ran in parallel to 2 nd study to confirm robustness results. The red stars indicate cases when performance decline was rectified by maintenance of UHPLC or simply transfer tube cleaning.

Further experimental details are presented in Section 2.

Figure S-6. Peak area stability over the course of experiments shown in Figure 2 for selected compounds.

Figure S-7. Quantitation of a) Ametryn at $1 \mathrm{ppb}$ (with RSD 4.2\%) and b) Fenazaquin at $0.5 \mathrm{ppb}$ (with RSD $2.4 \%$ ) as two examples from the pesticide analysis experiment shown in Figure 5.

First panel in each row shows chromatogram of the precursor ion, the second- library matching of that ion, and third panel shows the confirmation of the fragments. Fourth panel shows a calibration curve created for quantitation of that compound during method development.

Figure S-8. An example of peptide fragmentation on the Orbitrap Exploris 240 MS vs. Orbitrap Exploris $480 \mathrm{MS}$ at normalized collision energy (NCE) of 27. MS/MS spectral patterns (a) are virtually identical with similar fragment intensities across the entire mass range (b) and dot product of 0.98 .

Figure S-9. Dramatic decrease of fragmentation for mild trapping mode for selected compounds in an 18-min LC/MS run. The graph shows the ratio of peak area of fragment ion vs. precursor ion in percent with and without mild trapping mode settings applied.

Figure S-10. Sequence coverage maps for sub-units of trastuzumab (Figure 10) obtained using BioPharma Finder software at resolution setting 240,000. HCD fragmentation in IRM at collision energies $12,16,20 \mathrm{~V}$ was employed to maximize cleavages of all sub-units.

Figure S-11. RP LC/MS analysis of trastuzumab light and heavy chain subunits after the complete reduction of disulfide bonds. A monoisotopic mass for the light chain (a) and average masses for the 
glycoforms of the heavy chain (b) were obtained with low-ppm mass accuracy following deconvolution of the corresponding full scan spectra of the light (c) and the heavy chain (e), with chromatogram shown in (d). A high-resolution setting of 240,000 was used for the light chain and then switched to a low resolution of 15,000 during heavy chain elution. Isotopic resolution of the heavy chain was achieved in selected ion monitoring mode at 480,000 resolution setting (f).

Subsequent top-down analysis using HCD fragmentation in the IRM at different collision energies resulted in cleavage of $62 \%$ residues in the light chain and $33 \%$ residues in the heavy chain.

Figure S-12. Reproducibility of FAIMS-DDA proteomic analysis with the Orbitrap Exploris 480 MS for 5 replicate runs:

a) the number of identified peptide groups (mean 64,311 with $2 \% \mathrm{CV}$ ) and protein groups (mean 6,703 with $0.9 \% \mathrm{CV}$ ),

b) elution profiles for each replicate.

In each experiment, $200 \mathrm{ng}$ Pierce HeLa digest was analyzed with a 120 min LC gradient using a Top20 DDA method and all other experimental conditions identical to those in Figure 9.

Figure S-13. Reproducibility of DDA proteomic analysis with the Orbitrap Exploris 240 MS for 4 different instruments in 3 different locations worldwide:

a) the number of unique protein groups identified for 4 different instruments with 3 replicates each $(5,379$ mean with $2.6 \% \mathrm{CV})$,

b) the number of unique peptides identified for 4 different instruments with 3 replicates each (49,521 mean with $5.9 \% \mathrm{CV})$.

In each experiment $1000 \mathrm{ng}$ Pierce HeLa digest was analyzed with a 60 min LC gradient using a Top20 DDA method and EASY-Spray ES803A column.

Figure S-14. Quantitative comparison between replicates of FAIMS-DDA proteomic analysis with the Orbitrap Exploris $240 \mathrm{MS}$ for different sample loads shown in Figure 9:

a) correlation of datasets from 10 to $1000 \mathrm{ng}$ sample analysis using same LC/MS/MS method;

b) precision and accuracy of quantitation over 100x range of peptide loads, with the expected ratios indicated by black diamonds. 
Fig. S-1. a)

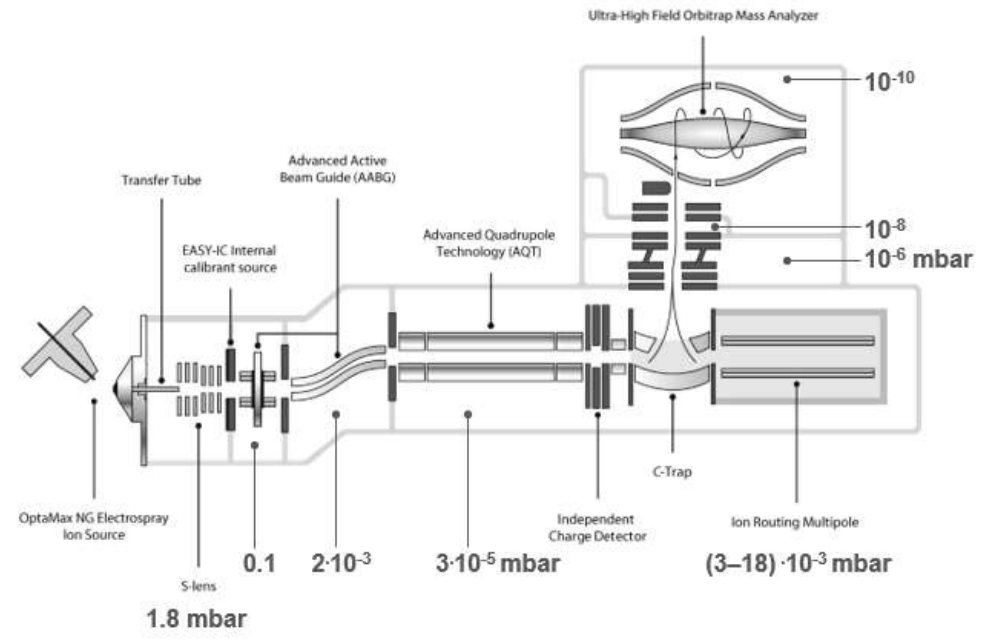

Fig. S-1. b)

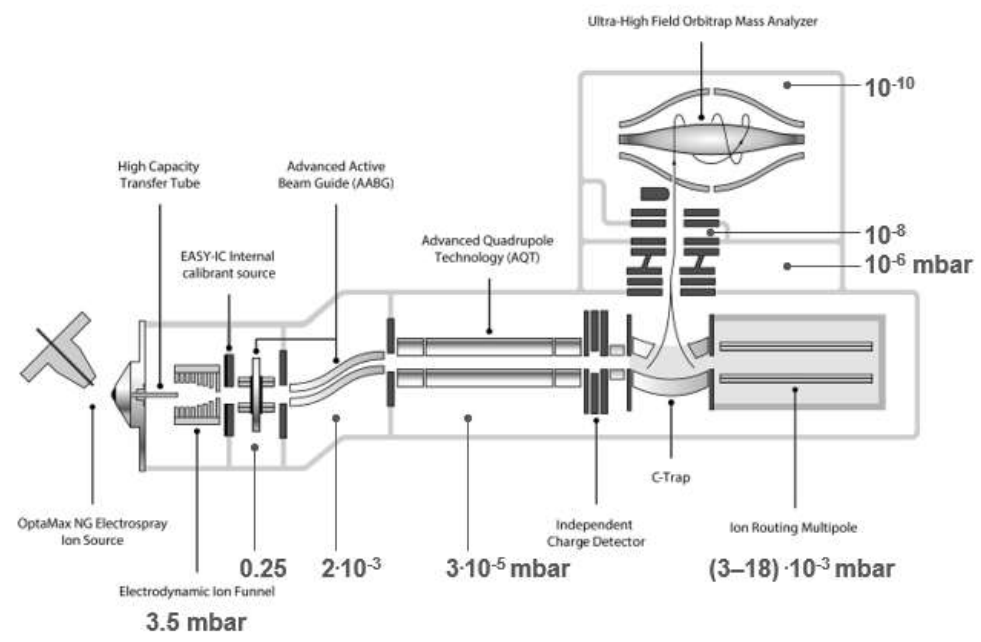


Fig. S-2

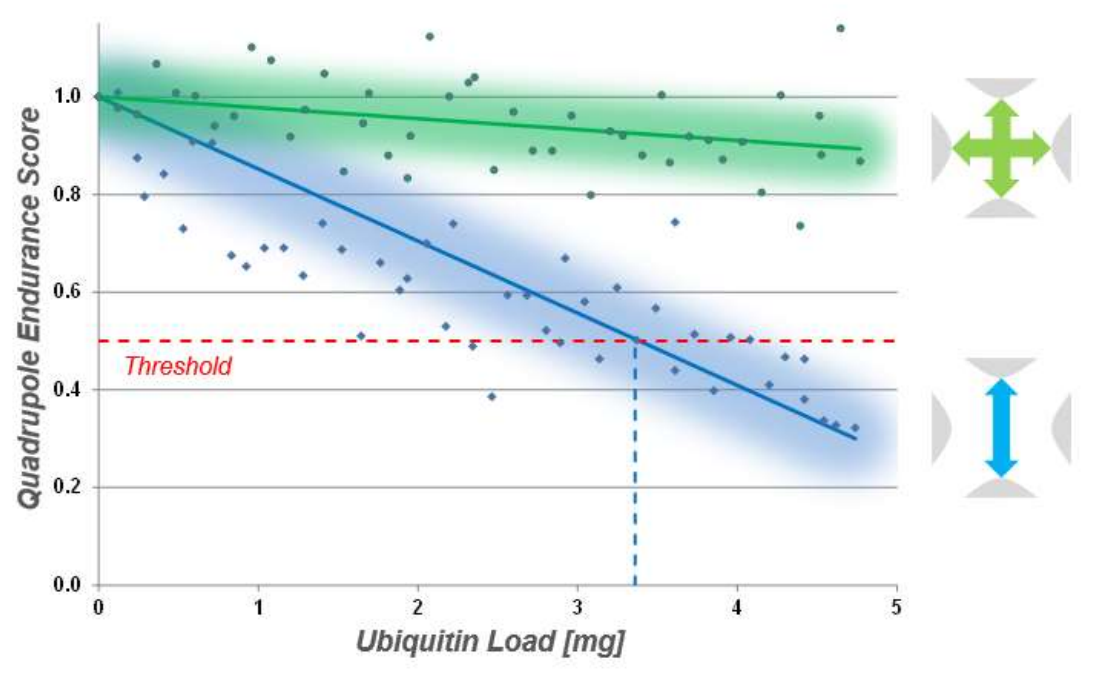

a)
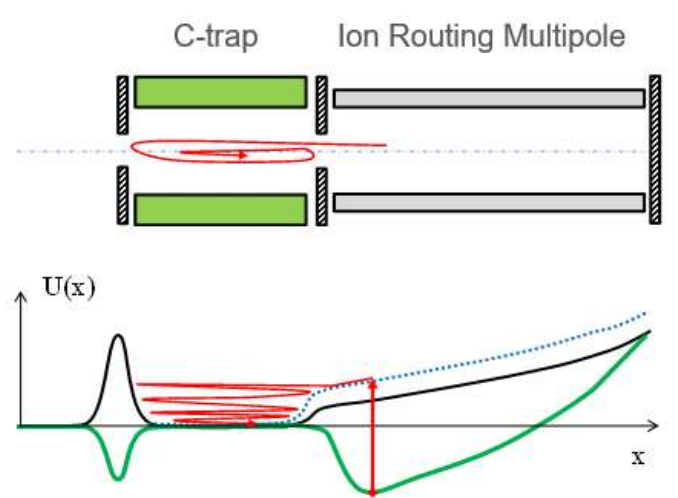

Fig. S-3 b)
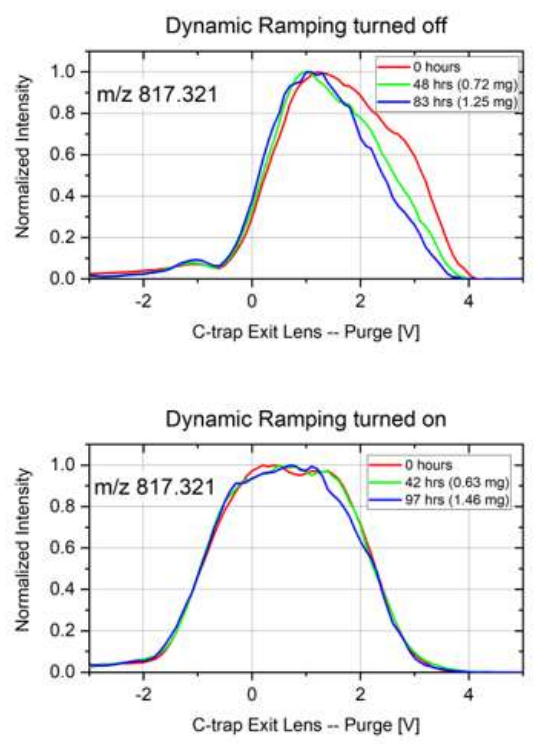
Fig. S-4

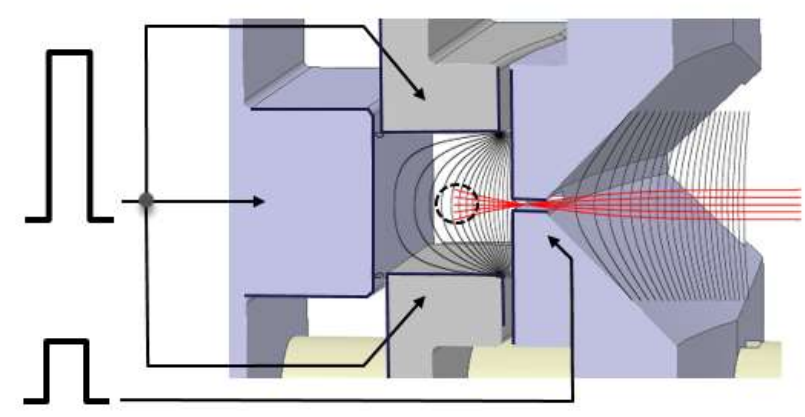

Fig S-5a:

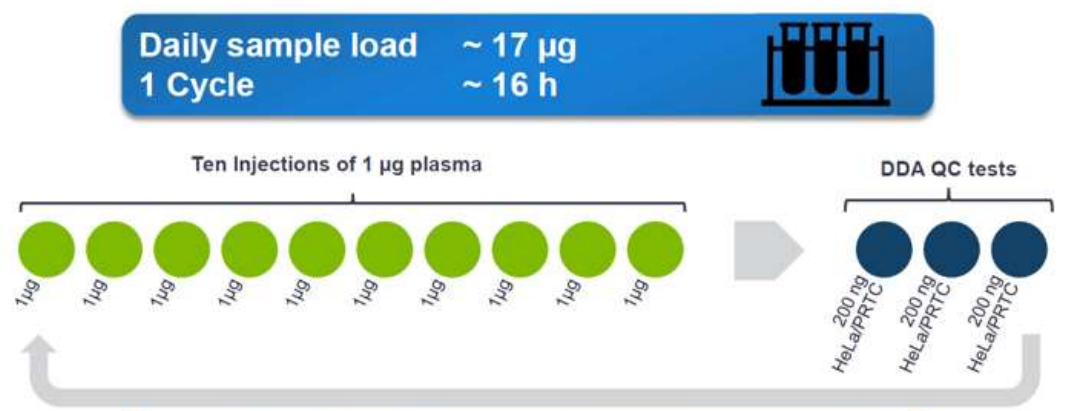

Repeat until HeLa/PRTC QC runs show

$>10 \%$ decline or system diagnostics fail 
Fig S-5b:
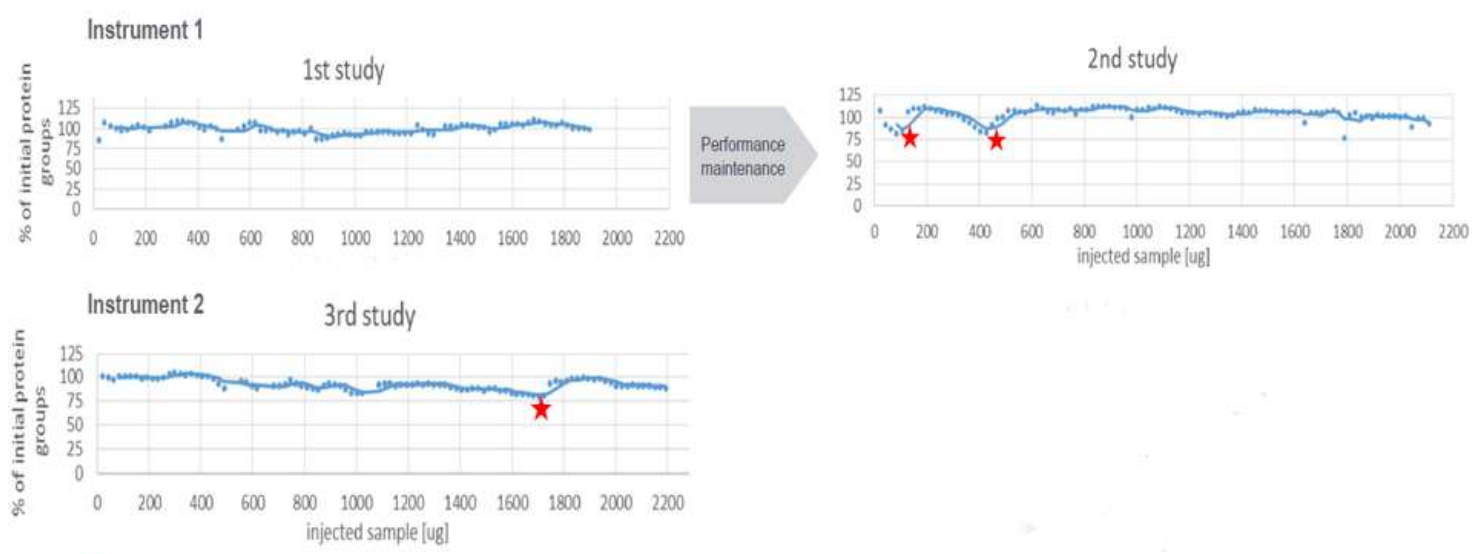

$\star$ performance decline recovered by $\mathrm{nLC}$ maintenance or tranfer tube cleaning

Figure S-6.

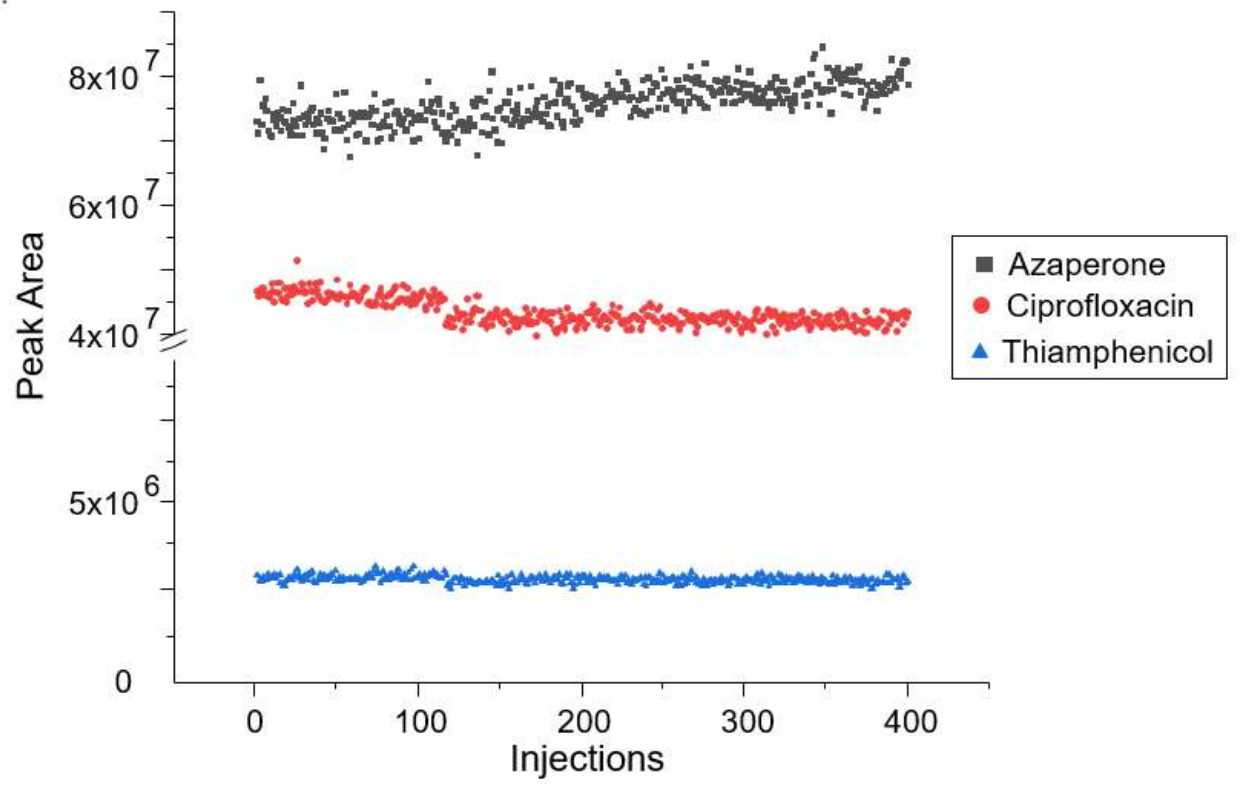


Figure S-7

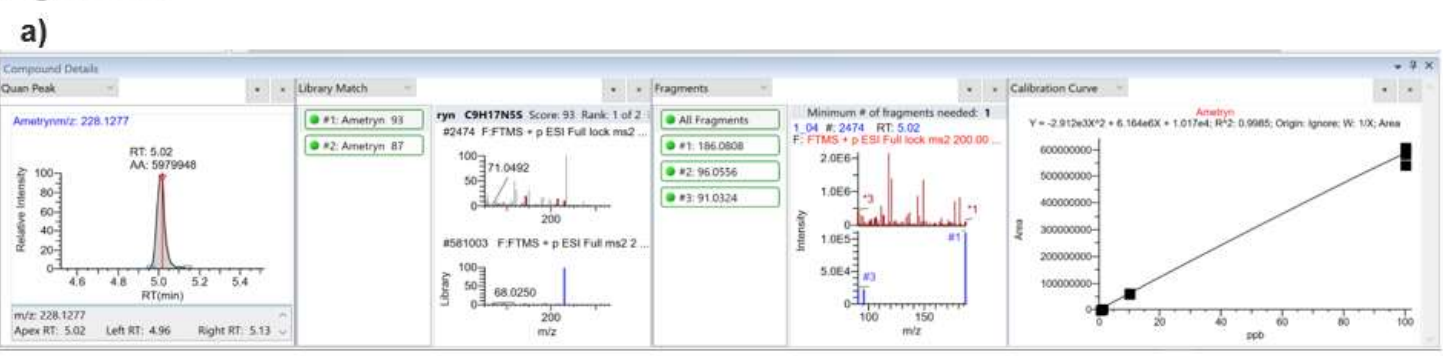

b)

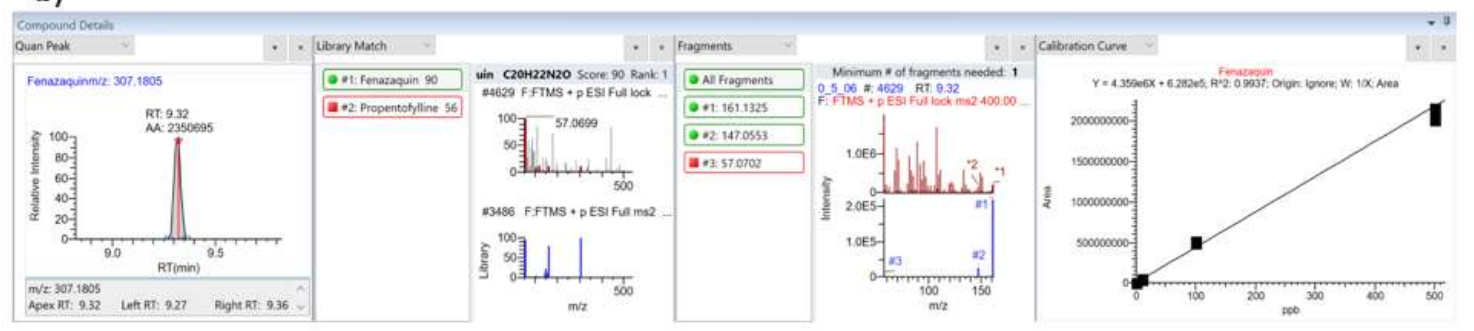

Figure S-8.

a)

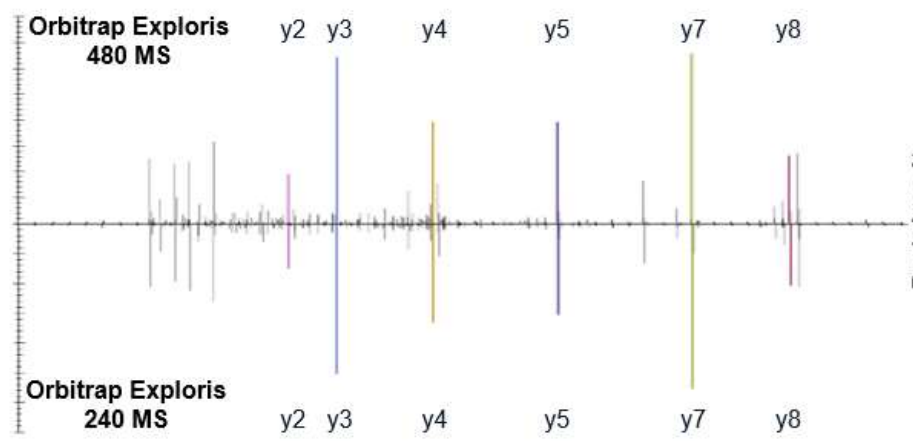

b) AKT1_HUMAN, NDGTFIGYK, m/z $511.7553 @$ NCE 27

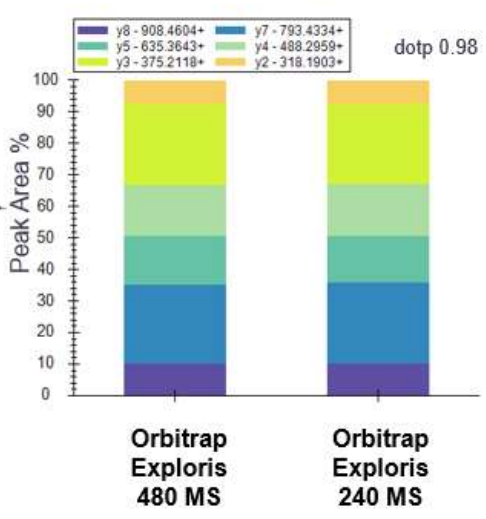


Figure S-9. Mild trapping

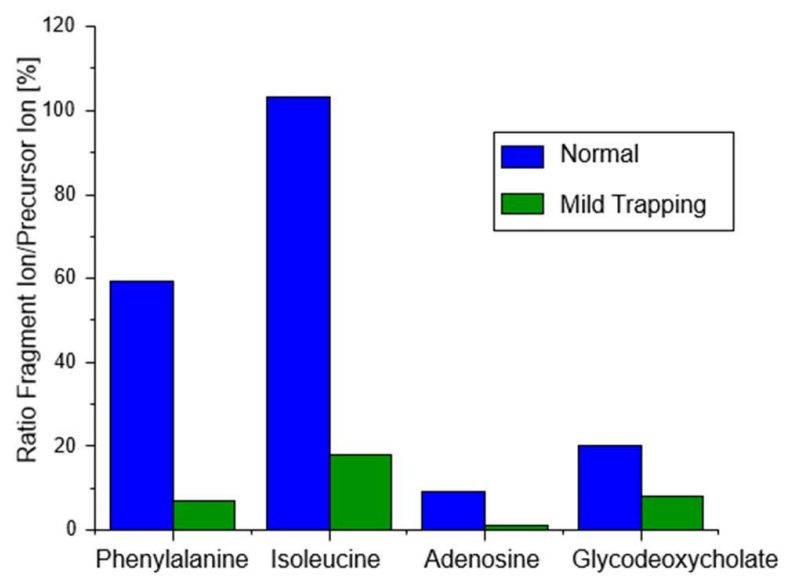

Figure S-10

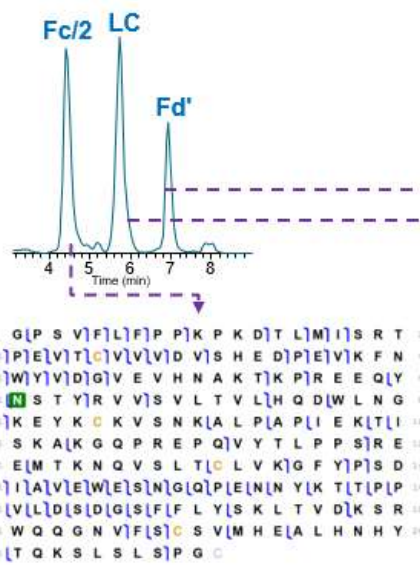

$\mathrm{Fc} / 2: 42 \%$ residue cleavages

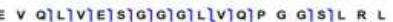
SATA]STG FN I K D TIYIII HW V R OIA A DIS VKGRF TIS A DITISIK N TIA]Y [L Q)M N]S L[R]A E D T|A]V|Y|Y]C[S]R]W]G

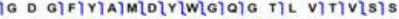
A $S T(K[O P S$ VLF]P LLA]PLS]S KS $S$ T $S O$ LO TLALA LLO L L VIK DLYIFLPLELPLVLT VIS IWLNLSLGLA LITLSLGIV H T F P A VLL alsLS

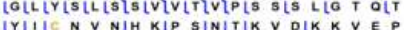
IYLILC N V NLH KLP SLNLTLK V DLK K V E P
$K S$ S D KLT H TIC PLPLCLP ALPELLL G

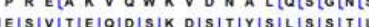
LLIS KLA DIY ELKLHIKLVIY A]CLEIV TIHIOLO

Fd': $52 \%$ residue cleavages 
Figure S-11.
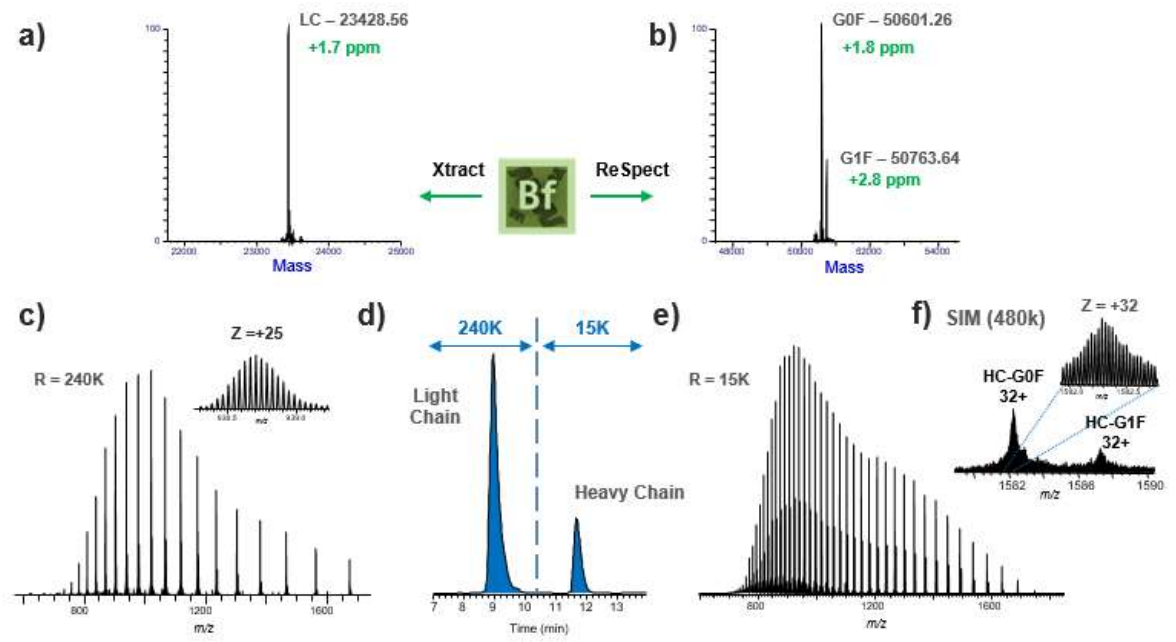

Figure S-12.

a)

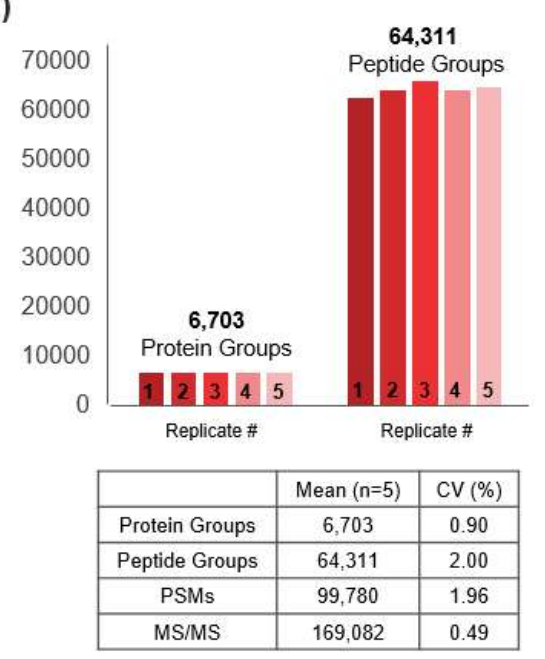

b)

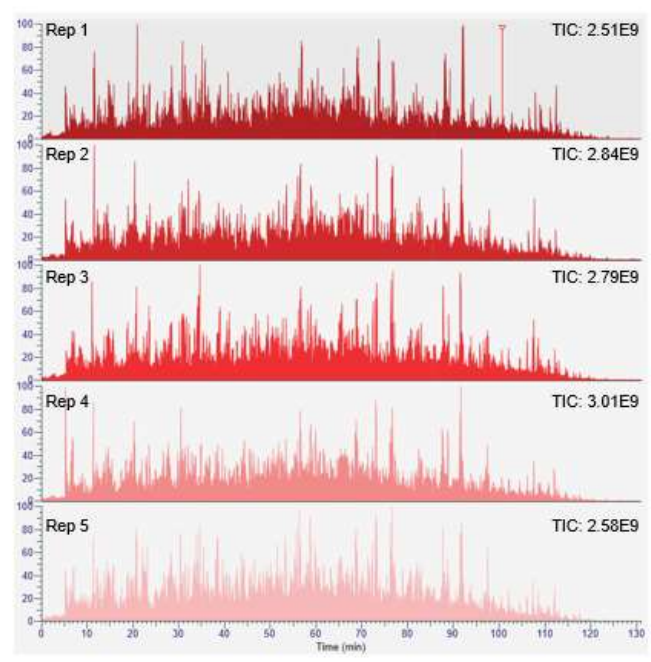


Figure S-13.

a)

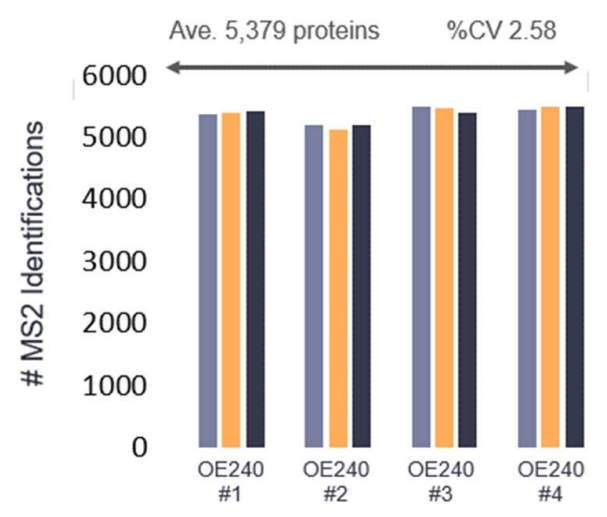

Figure S-14.

a)

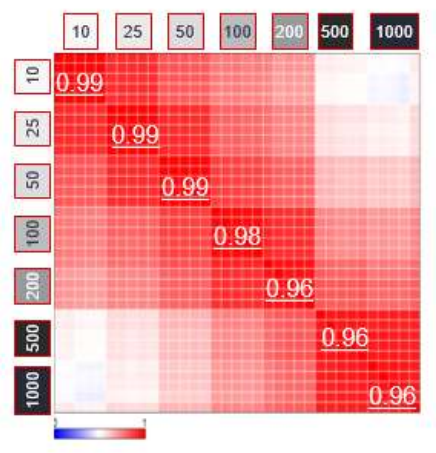

b)

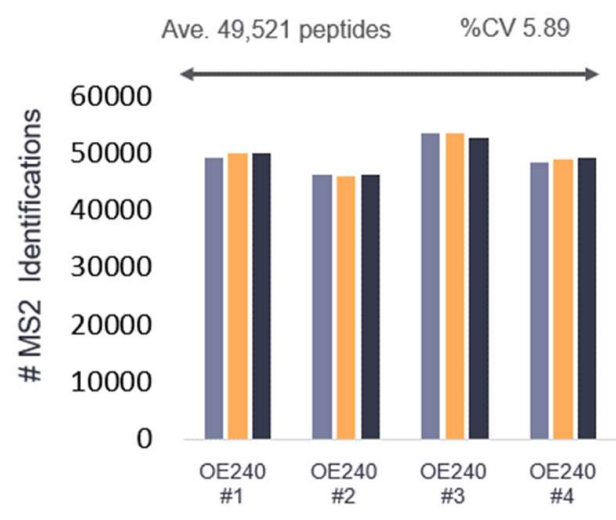

b)

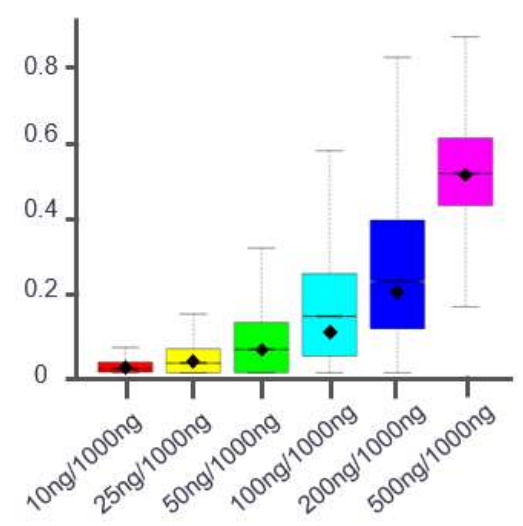

$\begin{array}{llllll}\text { Expected Ratio } 0.01 & 0.025 & 0.05 & 0.1 & 0.2 & 0.5\end{array}$

$\begin{array}{llllllll}\text { Actual Ratio } & 0.012 & 0.025 & 0.057 & 0.14 & 0.23 & 0.52\end{array}$ 